Edith Cowan University

Research Online

Research outputs 2014 to 2021

$12-9-2019$

\title{
Suboptimal health pregnant women are associated with increased oxidative stress and unbalanced pro- and antiangiogenic growth mediators: A cross-sectional study in a Ghanaian population
}

\author{
Enoch Odame Anto \\ Edith Cowan University \\ Peter Roberts \\ Edith Cowan University \\ David Anthony Coall \\ Edith Cowan University \\ Eric Adua \\ Edith Cowan University \\ Cornelius Archer Turpin
}

See next page for additional authors

Follow this and additional works at: https://ro.ecu.edu.au/ecuworkspost2013

Part of the Medicine and Health Sciences Commons

10.1080/10715762.2019.1685668

This is an Accepted Manuscript of an article published by Taylor \& Francis in Free Radical Research on 9 December 2019, available online: http://www.tandfonline.com/10.1080/10715762.2019.1685668.

Anto, E. O., Roberts, P., Coall, D. A., Adua, E., Turpin, C. A., Tawiah, A., ... \& Wang, W. (2020). Suboptimal health pregnant women are associated with increased oxidative stress and unbalanced pro-and antiangiogenic growth mediators: A cross-sectional study in a Ghanaian population. Free Radical Research, 54(1), 27-42. https://doi.org/ 10.1080/10715762.2019.1685668

This Journal Article is posted at Research Online.

https://ro.ecu.edu.au/ecuworkspost2013/7670 


\section{Authors}

Enoch Odame Anto, Peter Roberts, David Anthony Coall, Eric Adua, Cornelius Archer Turpin, Augustine Tawiah, Youxin Wang, and Wei Wang 

study in a Ghanaian population

4

$5 \quad{ }^{1,3}$ Enoch Odame Anto, ${ }^{1}$ Peter Roberts, ${ }^{1}$ David Coall, ${ }^{1}$ Eric Adua, ${ }^{2}$ Cornelius Archer Turpin, $6 \quad{ }^{2}$ Augustine Tawiah, ${ }^{4}$ Youxin Wang, ${ }^{1,5}$ Wei Wang*

$7 \quad{ }^{1}$ School of Medical and Health Sciences, Edith Cowan University, Perth, Western Australia

$8{ }^{2}$ Department of Obstetrics and Gynaecology, Komfo Anokye Teaching Hospital, Kumasi,

9 Ghana, West-Africa

$10{ }^{3}$ Department of Molecular Medicine, Kwame Nkrumah University of Science and 11 Technology, Kumasi, Ghana, West-Africa

${ }^{4}$ Beijing Key Laboratory of Clinical Epidemiology, School of Public Health, Capital Medical University, Beijing, China

${ }^{5}$ School of Public Health, Taishan Medical University, Taian, China

*Address correspondence to:

1) Professor, Wei Wang, MD, PhD, FFPH, FRSB, FRSM

School of Medical and Health Sciences, Edith Cowan University

270 Joondalup Drive, Perth, WA 6027, Australia

Tel: (61 8) 6304 3717; Fax: (61 8) 63042626

E-mail: wei.wang@ecu.edu.au

Orcid: 0000-0002-1430-1360 
Abstract

Optimal oxidative stress (OS) is important throughout pregnancy; however, an increased OS may alter placental angiogenesis culminating in an imbalanced of angiogenic growth mediators (AGMs). Suboptimal Health Status (SHS), a physical state between health and disease, may be associated with increased OS and unbalanced AGMs. In this study, we explored the association between SHS, biomarkers of OS (BOS) and AGMs among normotensive pregnant women (NTN-PW) in a Ghanaian Suboptimal Health Cohort Study (GHOACS). This comparative GHOACS recruited 593 NTN-PW from the Komfo Anokye Teaching Hospital, Ghana. SHS was measured using a Suboptimal Health Status Questionnaire-25 (SHSQ-25). Along with the subjective SHS measure, objective BOS: 8-hydroxy-2-deoxyguanosine (8-OHdG), 8-epiprostaglandinF2 alpha (8-epiPGF2 $\alpha$ ), total antioxidant capacity (TAC), and AGMs: vascular endothelial growth factor-A (VEGF-A), soluble fms-like tyrosine kinase receptor 1 (sFlt-1), placenta growth factor (PIGF) and soluble endoglin (sEng) were evaluated. Compared to optimal health NTN-PW, levels of PIGF, VEGF-A and TAC were significantly $(p<0.05)$ reduced and negatively associated with SHS whilst sEng, sFlt-1, 8-epiPGF2 $\alpha, 8-O H d G$, and combined ratios of sFlt-1/PlGF, 8-epiPGF2 $\alpha / \mathrm{PlGF}$, 8-OHdG/PlGF, and sEng/PlGF were significantly increased and positively associated with SHS. The 1st quartile for PIGF (2.79-fold) and VEGF-A (5.35-fold), and the 4th quartile for sEng (4.31-fold), sFlt1(1.84-fold), 8-epiPGF2 $\alpha$ (2.23-fold), 8-OHdG (1.90-fold) and urinary 8-OHdG (1.95fold) were independently associated with SHS $(p<0.05)$. SHS is associated with increased OS and unbalanced AGMs. Early identification of SHS-related OS and unbalanced AGMs may inform clinicians of the need for therapeutic options. 
Key words: pregnant women, oxidative stress, angiogenic growth mediators, suboptimal health status

\section{Introduction}

In recent years, a number of normotensive pregnant mothers, particularly in subSaharan African (SSA) countries continue suffer health complaints without diagnosable conditions, and this has led to increased morbidity and mortality rates $[1,2]$. Particularly, the stressful demands during pregnancy may alter physiological and metabolic functions and lead to health complaints including high-oxygen requirement and high-energy demand [3]. Although these dramatic events occur to sustain the mother and the growing foetus, they may also culminate in oxidative stress and adverse pregnancy outcomes such as stillbirth, intrauterine growth restriction and preterm delivery among others $[4,5]$.

Oxidative stress (OS) is an imbalance between pro-oxidant and anti-oxidant capacity [6]. Meanwhile, an optimal OS and reactive oxygen species (ROS) are essential throughout pregnancy to regulate a successful placental angiogenesis; a process whereby new blood vessels are formed from pre-existing ones during vascular development $[7,8]$. In an optimal OS state during placental angiogenesis and maternal vascular remodeling, the extravillous cytotrophoblast (EVT) cells of foetal origin invade the maternal uterine spiral arteries $[7,9]$. The resultant is the formation of a large capacity conduit vessel network which allows adequate exchange of blood and nutrient between the mother and the foetus [10]. During this process, the invasive EVT expresses a number of angiogenic growth mediators (AGMs) including pro-angiogenic growth factors such as VEGF-A and PlGF, and anti-angiogenic factor like sFlt-1 [7]. Both VEGF-A and PIGF are involved in angiogenesis, placental vascular remodeling, 
vascular permeability, nitric oxide (NO) production, promoting endothelial cell control and proliferation $[7,8]$.

Meanwhile, increased OS can be detrimental [6-8]. For instance, in an increased OS state, the EVT overexpresses sFlt-1, which antagonises the function of VEGF and PlGF on the endothelial cells leading to endothelial dysfunction [6,7]. Soluble endoglin (sEng), another anti-angiogenic growth factor which is highly expressed on the endothelial cells and cell membrane of the syncytiotrophoblast cells also antagonises the function of transforming growth factor beta 1 (TGF $\beta 1$ ), resulting in a loss of endothelial cell control, vasoconstriction and increased OS [7,9]. The increased OS may be caused by placental hypoxia/ischaemia originating from an incomplete maternal vascular remodeling $[7,8]$.

Several other factors including advanced maternal age, increased inflammatory response, cardiovascular diseases and hormonal changes contribute to increased ROS formation and OS in the circulation [10,11]. Particularly, an altered hormonal function during pregnancy is associated with elevated phospholipid levels/phospholipid accumulation [12]. Subsequently, increased levels of phospholipids at sites where ROS are formed lead to endogenous ROS-induced lipid peroxidation [13]. In addition, increased ROS formation in circulation can cause damage to proteins and DNA and lead to protein oxidation and oxidative DNA damage, respectively $[7,8]$. Biomarkers of oxidative stress (BOS) 8-epiPGF2 $\alpha$, and 8-OHdG are formed by free radical-catalysed phospholipid peroxidation and are potent markers indicative of in-vivo OS and oxidative DNA damage, respectively [13]. A compromised antioxidant system on the other hand, depicts a correspondingly reduced level of TAC [10].

Previous studies have extensively focused on increased levels of OS and imbalance in AGMs among women with complicated pregnancies [5,14] while paying 
less attention to these changes in normal pregnancies [15]. In addition to the dearth of data on evaluation of BOS and AGMs together in normal pregnancy, previous studies evaluated these markers in third trimester while paying less attention to these levels in the early trimesters of pregnancy. Early identification of increase OS and unbalanced levels of AGMs would improve diagnosis and treatment. Despite the fact that BOS and AGMs are sensitive and dynamic in both pregnancy and neonatal medicine, they are not used in routine antenatal care because they are expensive, invasive, requires a long turnaround time and expertise, and may not be readily available to women who visit under resourced hospitals. In addition, a longer turnaround time leads to delayed therapeutic interventions. An attempt to overcome this over the past few years has been the need to shift from reactive medical intervention to predictive, preventive and personalised medicine (PPPM) [16-20]. The approach of PPPM has adopted traditional, behavioural and environmental factors for early treatment and prevention of unrecognised diseases [20]. One way to identify participants with preconditions even before the onset of clinical manifestations, is to evaluate their physiological metrics at the preclinical or suboptimal health stage [19].

From the public health perspective, a recent development in the research for a promising suboptimal health status (SHS) evaluation measure that can be used in PPPM, is the development of a 25-question item Suboptimal Health Status Questionnaire (SHSQ-25). It is a subjective and non-invasive health assessment tool which is inexpensive, and requires less expertise and turnaround time. The SHSQ-25 was first created by our team and the term 'suboptimal health status' (SHS) was coined to define a physical state between health and disease [21,22]. SHS is recognised as a subclinical, reversible stage of chronic disease and characterised by poor health, low energy or vitality and general body weakness $[19,21,22]$. SHSQ-25 has since been used to evaluate SHS in 
several studies and was found useful for early detection and risk stratification of several symptoms and diseases [19,23-29]. For example, SHS was found to be an independent risk factor for type II diabetes mellitus in an African population [23], arterial stiffness and cardiovascular disease in European population [24], type II diabetes mellitus [25], cardiovascular diseases [26,27], psychosocial stress [28], and telomere length [29] in an Asian population.

Even though previous studies have reported a correlation of SHS with cardiovascular disease and arterial stiffness, which are both risk factors for increased oxidative stress, no study to date has explored together, its relationship with BOS and AGMs in pregnancy. Although OS and imbalance in AGMs are common in complicated pregnancies like preeclampsia, it is possible that SHS may precede its clinical manifestation. Our ongoing cohort study found that SHS is an independent measure for preeclampsia [30]. As a result, there is the need to evaluate if our NTN-PW experiencing suboptimal health exhibit a variation in OS and AGMs levels compared to optimal health status NTN-PW. For the first time in the present study, we explore an association of SHS with BOS and AGMs among normotensive pregnant women at 1020 weeks gestation in a Ghanaian Suboptimal Health Cohort Study (GHOACS). An increased OS and unbalanced AGMs, if found associated with SHS, would validate the usefulness of SHSQ-25 thereby creating a possibility to inform clinicians the need for early therapeutic options.

\section{Materials and Methods}

\section{Study design and participants}

As a part of the on-going Ghanaian Suboptimal Health Cohort Study (GHOACS), this hospital-based comparative cross-sectional study included 593 normotensive pregnant 
women (NTN-PW) attending regular antenatal care at the Obstetrics and Gynaecology

157 Department of Komfo Anokye Teaching Hospital (KATH), Kumasi Ghana. Both nulliparous and multiparous NTN-PW aged from 18 to 45 years with a singleton pregnancy from 10 to 20 weeks gestation gave written informed consent and were included in the present study. All participants were physically examined by a qualified consultant obstetrician/gynaecologist. The normotensive pregnancy was classified as pregnancy without measurable proteinuria and had normal blood pressure $(<140 / 90$ $\mathrm{mmHg}$ ) on two occasions at least four hours apart and had no history of a clinically diagnosed condition during the three months prior to the start of the present study. Exclusion criteria were women of advanced maternal age (>45 years), those below 18 years, multiple pregnancies, previous clinically known conditions such as preeclampsia, gestational diabetes, gestational hypertension, sexually transmitted infections, sickle cell anaemia, obesity and any form of clinically diagnosed cardiovascular condition. Also, those with current or previous history of smoking and alcoholic beverage intake at the time of sampling were excluded.

\section{Ethical consideration}

172 This study was approved by the Committee on Human Research Publication and Ethics

173 (CHRPE) of the School of Medical Science (SMS) /KNUST and Research and Development Unit, Komfo Anokye Teaching Hospital (KATH) (CHRPE/AP/146/17) and the Human Research Ethics Committee (HREC) of Edith Cowan University (ECU) (17509). Written informed consent in the form of signature and fingerprint was obtained

177 from participants and Legally Authorised Representatives before the start of the present 178 study. This study was conducted in accordance with the guidelines of the Helsinki 179 Declaration. 

data

The overall SHS of NTN-PW was assessed using SHSQ-25. The SHSQ-25 consist of five subclasses namely: fatigue ( 9 question item), cardiovascular system (3 question item), digestive system (3 question item), immune system (3 question item) and mental health (7 question item) $[19,22,31]$. These questions were explained to each participant in the native language by the consultant obstetrician/gynaecologist and their response were translated into English. Each pregnant woman was asked to rate her health statement on a 5-point Likert scale: never or almost never (1), occasionally (2), often (3), very often (4) and always (5) based on how often they had experienced a particular health complaint in the past 3 months. The raw scores of 1 to 5 were recoded as 0 to 4 for each participant followed by a summation of the codes for the 25 answered questions. The median of the total score was recorded as the cut-off point and values $\geq$ the median represented 'SHS' (poor health) and those $<$ indicated 'optimal health status (OHS)' $[19,22,31]$. In the present study, a score $\geq 19$ depicted SHS and $<19$ depicted OHS. A reliability test was performed on the SHSQ-25 and a Cronbach's alpha coefficient value was found to be 0.95 .

Sociodemographic, clinical and obstetric data were obtained from the antenatal folder and participant's record in the database of the KATH. Double measurements of blood pressure (BP) as well as weight, height and body mass index (BMI) were performed by trained personnel and midwives and values were recorded. The last BMI before conception (pre-gestation BMI) was also obtained from participants' records. 


\section{Biospecimen collection}

Participants provided 10-20 millilitre midstream urine samples in sterile leakproof containers. Dipstick proteinuria was determined for each participant. Samples were centrifuged at $3000 \mathrm{rpm}$ for 10 minutes at $4{ }^{\circ} \mathrm{C}$ (HERMLE® Z306K, Wehingen, Germany) and the supernatants were aliquoted into two cryovials tubes ( $1 \mathrm{ml}$ each). One millilitre of the aliquot was used to measure urine creatinine $(\mathrm{Cr})$ concentrations and the rest were stored at $-80{ }^{\circ} \mathrm{C}$ (Thermo scientific ultra-low freezer) until further analysis. An overnight fasting venous blood sample (10 millilitres) were collected between $8 \mathrm{am}$ and 11 am from each of the 593 participants and were dispensed into specialised vacutainer ${ }^{\circledR}$ tubes. The serum and plasma were obtained following centrifugation at $3000 \mathrm{rpm}$ for 10 minutes and were separated into two cryovials each and stored at $-80{ }^{\circ} \mathrm{C}$ (Thermo scientific ultra-low freezer) until assay.

\section{Haematobiochemical assay}

Plasma fasting blood glucose (FBG), serum triglyceride (TG), total cholesterol

(TC), high density lipoprotein cholesterol (HDL-c), low density lipoprotein cholesterol (LDL-c), alanine aminotransferase (ALT), aspartate aminotransferase (AST), gamma glutamyl transferase (GGT), total protein (TP), albumin (ALB), lactate dehydrogenase (LDH), alkaline phosphatase (ALP), urea, creatinine (Cr), uric acid (UA), sodium (Na), potassium $(\mathrm{K})$, chloride $(\mathrm{Cl}-)$, magnesium $(\mathrm{Mg})$ and calcium $(\mathrm{Ca})$ were measured using an automatic chemistry analyser (Roche Diagnostics, COBAS INTEGRA 400 Plus, USA). Haemoglobin, red blood cell distribution width (RDW) and platelet count (PLT) were analysed using a Mindray Haematology Analyzer BC 2800. 


\section{Angiogenic growth mediator (AGMs) assay}

Serum concentrations of VEGF-A, sFlt-1, PIGF, and sEng were measured in duplicate using competitive ELISA kits from R\&D System Inc. (Minneapolis, MN USA). Absorbance was measured at $450 \mathrm{~nm}$ wavelength using a microplate ELISA reader (Bio-Tek ELx808 microplate reader, Hayward, CA, USA). The concentrations of each biomarker were derived from standard curves from a known standard concentration of recombinant factors.

\section{Biomarkers of oxidative stress (BOS) assay}

Following the manufacturer's instructions, urinary and serum 8-OHdG were analysed in duplicates using highly sensitive and competitive ELISA kits (ab201734, Abcam, China). Serum concentrations were determined by comparison to a standard curve and recorded in ng/L. The inter-and-intra assay coefficients of variation (CV) were $3.5 \%$ and $4.5 \%$, respectively. Urinary $8-\mathrm{OHdG}$ concentrations obtained from the standard curves were normalised to creatinine concentrations and recorded as $\mathrm{ng} / \mathrm{mg} \mathrm{Cr}$.

Serum 8-epi-PGF2 $\alpha$ was analysed in duplicate using competitive ELISA kits from ELabscience, China (cat. LogE-EL-0041). The intra-and-inter assay coefficients of variation $(\mathrm{CV})$ were $5.6 \%$ and $6.4 \%$, respectively. The absorbance of both 8 -epi-PGF2 $\alpha$ and $8-\mathrm{OHdG}$ was read at 450nm on a microplate reader (Bio-Tek ELx808 microplate reader, Hayward, CA, USA).

TAC reagents were obtained from Sigma-Aldrich (Hong Kong, China). Plasma samples were thawed to measure TAC spectrophotometrically at $593 \mathrm{~nm}$ using Mindray BA-88A, China. The estimation of TAC was based on ferric reducing ability of plasma (FRAP) and the protocol as described by Benzie and Strain [32]. The absorbance was 
used to obtain the concentrations after comparison to standard curves and recorded in $\mu \mathrm{mol} / 1$.

\section{Statistical analysis}

254 Normalisation of the data was performed using Kolmogorov-Smirov test. Data was presented as mean $\pm \mathrm{SD}$ for parametric continuous variables, median (interquartile ranges) for non-parametric continuous variables and frequency (percentages) for categorical variables. Chi-square test was performed to test associations between categorical variables. The difference in mean variables between SHS and OHS was tested using an independent sample t-test. The difference in median variables between SHS and OHS was tested using the Mann Whitney U-test. A multivariate logistic regression model was performed to test risk factors associated with SHS. Linear regression models were performed to test the associations between SHS, AGMs and OS biomarkers. Data analysis was performed using R version 3.4.3 (R core Team 2017), SPSS version 24 (IBM Corp, NY, USA) and XLSTAT Premium version 2018.1 for windows. $P$ value $<0.05$ was considered statistically significant.

Results

\section{Sociodemographic characteristics of NTN-PW stratified as SHS and Optimal health status}

The average age of the participants was 29.64 years (Table 1). A higher proportion [34.6\% (205/593)] of the study participants were aged 25 to 30 years. There was no statistically significant difference between the mean ages of pregnant women with SHS compared to those with OHS $(29.44 \pm 5.92$ vs. $29.77 \pm 6.08 ; p=0.5045)$. Overall, a higher

272 proportion of the pregnant women had completed secondary education [40.8\% (242/593)], were married [84.5\% (501/593)], were Akan's by ethnicity [87.2\% (517/593)], had an informal occupation [63.2\% (375/593)] and earned a low-income per month [38.8\% 
275 (230/593)]. However, there was no statistically significant difference in proportion between

276 pregnant women with SHS compared to OHS in terms of level of education $(p=0.7577)$,

277 marital status $(p=0.7000)$, ethnicity $(p=0.9140)$, occupation $(p=0.7913)$ and basic monthly

278 salary income $(p=0.8384)$ (Table 1).

Table 1 Sociodemographic characteristics of NTN-PW stratified by SHS and OHS

\begin{tabular}{|c|c|c|c|c|c|}
\hline Characteristics & $\begin{array}{c}\text { Total } \\
(\mathrm{N}=593)\end{array}$ & $\begin{array}{c}\text { SHS } \\
(\mathrm{N}=297)\end{array}$ & $\begin{array}{c}\text { OHS } \\
(\mathrm{N}=296)\end{array}$ & Statistics & $p$-value \\
\hline Age (mean \pm SD) (years) & $29.64 \pm 5.98$ & $29.44 \pm 5.92$ & $29.77 \pm 6.08$ & 0.6678 & 0.5045 \\
\hline \multicolumn{6}{|l|}{ Age (years) } \\
\hline $18-24$ & $130(21.9)$ & $66(22.2)$ & $64(21.6)$ & & \\
\hline $25-30$ & 205(34.6) & $110(37.0)$ & $95(32.1)$ & & \\
\hline $31-34$ & $124(20.9)$ & $58(19.5)$ & $66(22.3)$ & & \\
\hline $35-45$ & $134(22.6)$ & $63(21.2)$ & 71(23.9) & & \\
\hline Highest Level of Education & & & & $1.180,3$ & 0.7577 \\
\hline Unschooled & $5(0.8)$ & $2(0.7)$ & $3(1.0)$ & & \\
\hline Primary & 203(34.2) & $100(33.7)$ & $103(34.8)$ & & \\
\hline Secondary & $242(40.8)$ & $127(21.4)$ & $115(38.9)$ & & \\
\hline Tertiary & $143(24.1)$ & $68(22.9)$ & $75(25.3)$ & & \\
\hline Marital Status & & & & $0.714,2$ & 0.7000 \\
\hline Never married & $86(14.5)$ & $42(14.1)$ & $44(14.9)$ & & \\
\hline Married & $501(84.5)$ & $251(84.5)$ & $250(84.5)$ & & \\
\hline Cohabiting & $6(1.0)$ & $4(1.3)$ & $2(0.7)$ & & \\
\hline Ethnicity & & & & $0.522,3$ & 0.9140 \\
\hline Akan & $517(87.2)$ & 273(91.9) & $244(82.4)$ & & \\
\hline Ga-Adangbe & $10(1.7)$ & $6(2.0)$ & $4(1.4)$ & & \\
\hline Mole Dagbani & $49(8.2)$ & $49(16.5)$ & $45(15.2)$ & & \\
\hline Ewe & $8(1.3)$ & $5(1.7)$ & $3(1.0)$ & & \\
\hline Occupation & & & & $0.468,2$ & 0.7913 \\
\hline Unemployed & $63(10.6)$ & $34(11.4)$ & $29(9.8)$ & & \\
\hline Formal & $155(26.1)$ & $78(26.3)$ & $77(26.0)$ & & \\
\hline Informal & $375(63.2)$ & $185(62.3)$ & $190(64.2)$ & & \\
\hline Basic monthly income (GHQ) & & & & $0.846,3$ & 0.8384 \\
\hline None & $63(10.6)$ & $34(11.4)$ & $29(9.8)$ & & \\
\hline Low $(<500.0)$ & $230(38.8)$ & $114(38.4)$ & $116(39.2)$ & & \\
\hline Middle (500.0-1000.0) & 198(33.4) & $101(34.0)$ & $97(32.8)$ & & \\
\hline High $(>1000.0)$ & $102(17.2)$ & $48(16.2)$ & $54(18.2)$ & & \\
\hline
\end{tabular}

Values are presented as frequency (proportion); mean \pm SD (standard deviation); GHC: Ghana cedi. Statistics is represented as Chi-square value, degree of freedom $\left(X^{2}, \mathrm{df}\right)$, and t-test value (italised) 
A higher proportion of pregnant women were nulliparous [39.6\% (235/593)],

287 primigravida [46.2\% (274/593)], had optimal blood pressure [60.4\% (358/593)] and were overweight at both pre-gestational [37.8\% (224/593)] and the time of sampling [38.6\% (229/593)] (Table 2). There was a statistically significant difference in proportion between pregnant women with SHS compared to OHS in terms of parity $(p=0.0311)$, gravidity ( $p$ $=0.0309)$, and BP $(p<0.0001)$. In comparison to pregnant women with OHS, those with SHS had higher proportions in terms of high BP $(11.4 \%$ vs. $2.0 \% ; p<0.0001)$, family history of hypertension $(23.2 \%$ vs. $7.1 \% ; p<0.0001)$ and history of spontaneous abortion $(37.0 \%$ vs. $28.0 \% ; p=0.0282$ ). However, there was no statistically significant difference in proportion between pregnant women with SHS compared to OHS in terms of previous caesarean section (19.5\% vs. $21.6 \% ; p=0.5436)$. Consequently, there was a statistically significant difference 297 in the mean systolic blood pressure (SBP) between pregnant women with SHS compared to OHS ( $p=0.0071$ ) but no significant difference in the mean diastolic blood pressure (DBP) ( $p$ $=0.1574)$, gestational age $(p=0.9515)$, pre-gestation BMI $(p=0.6855)$ and BMI at the time of sampling ( $p=0.7658$ ) between groups. There were significantly reduced levels of serum $\mathrm{Mg}$ ( $p<0.0001)$, Ca $(p<0.0001)$, haemoglobin $(p=0.0428)$ and HDL-c $(p=0.0481)$ but significantly elevated levels of AST ( $p<0.0001)$, ALT ( $p=0.0158)$ ALP $(p=0.0032)$, GGT $(p<0.0001)$, urea $(p=0.0242)$, creatinine $(p=0.0467)$, uric acid $(p=0.0002)$ and TG $(p$ $=0.0007)$ among participants with SHS compared to those with OHS (Table 2). stratified by SHS and OHS

\begin{tabular}{lc}
\hline Characteristics & Total $(\mathrm{N}=593)$ \\
\hline Parity
\end{tabular}
SHS $(\mathrm{N}=297)$ OHS (N=296) 


\begin{tabular}{|c|c|c|c|c|c|}
\hline Nulliparous (0) & 235(39.6) & $113(38.0)$ & $122(41.2)$ & & \\
\hline Primiparous (1) & $114(19.2)$ & $64(21.5)$ & $50(16.9)$ & & \\
\hline Multiparous (2-4) & $244(41.2)$ & $120(40.5)$ & $124(41.9)$ & & \\
\hline Gravidity & & & & $6.951,2$ & 0.0309 \\
\hline Primigravida (1) & $274(46.2)$ & $153(51.5)$ & $121(40.9)$ & & \\
\hline Multigravida (2-4) & $175(29.5)$ & $81(27.3)$ & $94(31.8)$ & & \\
\hline Grand multigravida $(>5)$ & $144(24.3)$ & $63(21.2)$ & $81(27.4)$ & & \\
\hline BP (mmHg) & & & & $54.65,2$ & $<0.0001$ \\
\hline Normal (120-129/80-84) & $553(93.3)$ & $263(88.5)$ & $290(98.0)$ & & \\
\hline High (130-139/85-89) & $40(6.7)$ & $34(11.4)$ & $6(2.0)$ & & \\
\hline FH of HTN (Yes) & $90(15.2)$ & $69(23.2)$ & $21(7.1)$ & $33.81,1$ & $<0.0001$ \\
\hline H. Spont. Abort. (Yes) & $193(32.5)$ & $110(37.0)$ & $83(28.0)$ & $5.083,1$ & 0.0282 \\
\hline Previous CS (Yes) & $122(20.6)$ & $58(19.5)$ & $64(21.6)$ & $0.397,1$ & 0.5436 \\
\hline Protein $(<0.3 g / g / 24 h r)$ & $593(100.0)$ & $297(100.0)$ & $276(100.0)$ & & 0.9991 \\
\hline GA (weeks) & $16.98 \pm 2.01$ & $16.97 \pm 2.08$ & $16.98 \pm 1.98$ & 0.061 & 0.9515 \\
\hline SBP (mmHg) & $114.7 \pm 10.57$ & $115.8 \pm 11.00$ & $113 \pm 10.01$ & 2.703 & 0.0071 \\
\hline DBP (mmHg) & $72.58 \pm 9.26$ & $73.12 \pm 9.31$ & $72.04 \pm 9.20$ & 1.416 & 0.1574 \\
\hline Pre-gest. BMI $\left(\mathrm{Kg} / \mathrm{m}^{2}\right)$ & $27.04 \pm 4.83$ & $26.65 \pm 4.74$ & $27.12 \pm 4.92$ & 0.405 & 0.6855 \\
\hline Gest. BMI (Kg/m²) & $27.33 \pm 4.81$ & $27.39 \pm 4.74$ & $27.12 \pm 4.92$ & 0.298 & 0.7658 \\
\hline $\mathrm{Mg}(\mathrm{mmol} / \mathrm{l})$ & $0.95 \pm 0.19$ & $0.91 \pm 0.24$ & $0.99 \pm 0.13$ & 5.384 & $<0.0001$ \\
\hline $\mathrm{Ca}(\mathrm{mmol} / \mathrm{l})$ & $2.18 \pm 0.35$ & $2.07 \pm 0.38$ & $2.29 \pm 0.27$ & 8.431 & $<0.0001$ \\
\hline $\mathrm{Na}(\mathrm{mmol} / \mathrm{l})$ & $136.3 \pm 2.00$ & $136.4 \pm 1.99$ & $136.2 \pm 2.01$ & 0.958 & 0.3384 \\
\hline $\mathrm{K}(\mathrm{mmol} / \mathrm{l})$ & $4.18 \pm 0.38$ & $4.21 \pm 0.45$ & $4.17 \pm 0.33$ & 1.195 & 0.2326 \\
\hline $\mathrm{Cl}-(\mathrm{mmol} / \mathrm{l})$ & $105.6 \pm 2.32$ & $105.5 \pm 2.31$ & $105.6 \pm 2.33$ & 0.399 & 0.6889 \\
\hline LDH (IU/L) & $173.3 \pm 41.25$ & $176.4 \pm 45.14$ & $170.1 \pm 36.73$ & 0.061 & 0.0605 \\
\hline AST (IU/L) & $15.70(13.70-20.50)$ & $16.10(13.80-26.15)$ & $15.20(13.60-19.30)$ & 3504 & $<0.0001$ \\
\hline ALT (IU/L) & $11.50(10.30-16.65)$ & $12.60(10.30-18.40)$ & $11.05(10.20-14.50)$ & 3893 & 0.0158 \\
\hline ALP (IU/L) & $201.0(168.0-228.0)$ & $205.0(168.0-235.0)$ & $195.0(168.0-218.0)$ & 3781 & 0.0032 \\
\hline GGT (IU/L) & $10.40(9.80-13.50)$ & $11.30(10.10-15.40)$ & $10.34(9.70-12.20)$ & 3204 & $<0.0001$ \\
\hline Total protein $(\mathrm{g} / \mathrm{L})$ & $67.98 \pm 2.21$ & $68.01 \pm 2.21$ & $67.96 \pm 2.20$ & 0.266 & 0.7900 \\
\hline Albumin (g/L) & $36.85 \pm 1.27$ & $36.88 \pm 1.26$ & $36.82 \pm 1.27$ & 0.556 & 0.5782 \\
\hline Urea (mmol/l) & $3.76 \pm 1.61$ & $3.92 \pm 1.79$ & $3.62 \pm 1.39$ & 2.260 & 0.0242 \\
\hline Creatinine $(\mu \mathrm{mol} / \mathrm{l})$ & $61.19 \pm 13.51$ & $62.29 \pm 15.17$ & $60.08 \pm 11.53$ & 1.995 & 0.0465 \\
\hline Uric acid $(\mu \mathrm{mol} / \mathrm{l})$ & $290.0 \pm 46.10$ & $297.0 \pm 42.54$ & $283.0 \pm 48.48$ & 3.748 & 0.0002 \\
\hline Haemoglobin (g/dL) & $11.65 \pm 0.60$ & $11.01 \pm 0.63$ & $11.69 \pm 0.57$ & 1.646 & 0.0428 \\
\hline RDW-CV (\%) & $13.65 \pm 1.25$ & $13.66 \pm 1.30$ & $13.65 \pm 1.19$ & 0.123 & 0.9022 \\
\hline PLT (X109 / L) & $296.9 \pm 86.75$ & $290.8 \pm 85.70$ & $303.0 \pm 87.51$ & 1.718 & 0.0864 \\
\hline FBG (mmol/L) & $5.09 \pm 0.74$ & $5.12 \pm 0.77$ & $5.08 \pm 0.69$ & 0.635 & 0.5085 \\
\hline TC (mmol/L) & $4.65 \pm 1.18$ & $4.69 \pm 1.23$ & $4.61 \pm 1.11$ & 0.827 & 0.4088 \\
\hline TG (mmol/L) & $1.31 \pm 0.68$ & $1.39 \pm 0.76$ & $1.24 \pm 0.58$ & 2.706 & 0.0070 \\
\hline HDL-c (mmol/L) & $1.45 \pm 0.32$ & $1.40 \pm 0.32$ & $1.48 \pm 0.34$ & 1.898 & 0.0481 \\
\hline LDL-c (mmol/L) & $2.79 \pm 1.05$ & $2.84 \pm 1.12$ & $2.74 \pm 0.98$ & 1.171 & 0.2421 \\
\hline
\end{tabular}


As shown in Table 3, after adjusting for confounding factors using a multivariate 319 logistic regression model, the association remained significant with high $\mathrm{BP}$ [aOR=5.96, 95\% 320 CI (2.39-14.85); $\mathrm{p}<0.0001]$, low $\mathrm{Mg}[\mathrm{aOR}=4.47,95 \%$ CI (3.16-10.15); $\mathrm{p}<0.0001]$, low $\mathrm{Ca}$ $321[\mathrm{aOR}=2.19,95 \% \mathrm{CI}(1.19-5.03), \mathrm{p}<0.0001]$, high $\mathrm{LDH}[\mathrm{aOR}=2.75(1.60-5.07), \mathrm{p}=0.0006]$, 322 high AST $[\mathrm{aOR}=2.22(1.68-8.14), \mathrm{p}=0.0018]$, high creatinine $[\mathrm{aOR}=3.15,95 \%$ CI $(1.55-$ 323 7.04), $\mathrm{p}=0.0028]$, anaemia $[\mathrm{aOR}=1.58,95 \% \mathrm{CI}(1.11-2.62), \mathrm{p}=0.0397]$, high $\mathrm{TG}[\mathrm{aOR}=2.14$, $32495 \%$ CI (1.08-4.79), $\mathrm{p}=0.0206]$ and low HDL-c [aOR=2.57, 95\% CI (1.15-7.05), $\mathrm{p}=0.0418]$ as independent risk factors for SHS.

326

327

328

329 


\begin{tabular}{|c|c|c|c|c|c|c|}
\hline \multirow[b]{2}{*}{ Characteristics } & \multirow[b]{2}{*}{ SHS } & \multirow[b]{2}{*}{ OHS } & \multirow{2}{*}{$\frac{\text { Model } 1}{\operatorname{cOR}(95 \% \mathrm{CI})}$} & \multirow[b]{2}{*}{$P$ value } & \multirow{2}{*}{$\frac{\text { Model } 2}{\operatorname{aOR}(95 \% \mathrm{CI})}$} & \multirow[b]{2}{*}{$P$ value } \\
\hline & & & & & & \\
\hline \multicolumn{7}{|l|}{ BP (mmHg) } \\
\hline Optimal Normal & 263(88.6) & $290(98.0)$ & 1.00 & & 1.00 & \\
\hline High & $34(11.4)$ & $6(2.0)$ & $6.6(2.74-15.96)$ & $<0.0001$ & $5.96(2.39-14.85)$ & $<0.0001$ \\
\hline \multicolumn{7}{|l|}{$\mathrm{Mg}(\mathrm{mmol} / \mathrm{l})$} \\
\hline Low & $50(16.8)$ & $8(2.7)$ & $5.28(3.37-11.67)$ & $<0.0001$ & $4.47(3.16-10.15)$ & $<0.0001$ \\
\hline Normal & $247(83.2)$ & $288(97.3)$ & 1.00 & & 1.00 & \\
\hline \multicolumn{7}{|c|}{ Alb. Adj. Ca (mmol/l) } \\
\hline Low & $159(53.5)$ & $70(23.6)$ & $2.72(1.61-5.29)$ & $<0.0001$ & $2.19(1.19-5.03)$ & $<0.0001$ \\
\hline Normal & $138(46.5)$ & $226(76.4)$ & 1.00 & & 1.00 & \\
\hline \multicolumn{7}{|l|}{ LDH (IU/L) } \\
\hline High & $50(16.8)$ & $20(6.8)$ & $2.79(1.62-4.82)$ & 0.0002 & $2.75(1.60-5.07)$ & 0.0006 \\
\hline Normal & $247(83.2)$ & $276(93.2)$ & 1.00 & & 1.00 & \\
\hline \multicolumn{7}{|l|}{ AST (IU/L) } \\
\hline High & $24(8.1)$ & $6(2.0)$ & $2.25(1.71-9.56)$ & 0.0011 & $2.22(1.68-8.14)$ & 0.0018 \\
\hline Normal & 273(91.9) & $290(98.0)$ & 1.00 & & 1.00 & \\
\hline \multicolumn{7}{|l|}{$\operatorname{ALP}(\mathbf{I U} / \mathbf{L})$} \\
\hline High & $82(27.6)$ & $79(26.7)$ & $1.04(0.72-1.50)$ & 0.8536 & $1.08(0.78-1.93)$ & 0.8054 \\
\hline Normal & $215(72.4)$ & $217(73.3)$ & 1.00 & & 1.00 & \\
\hline \multicolumn{7}{|l|}{ Urea (IU/L) } \\
\hline High & $12(4.0)$ & $4(1.4)$ & $3.07(0.97-9.64)$ & 0.0729 & $3.03(0.73-10.51)$ & 0.0910 \\
\hline Normal & $285(96.0)$ & 292(98.6) & 1.00 & & 1.00 & \\
\hline \multicolumn{7}{|c|}{ Creatinine (IU/L) } \\
\hline High & $32(10.8)$ & $11(3.7)$ & $3.12(1.54-6.33)$ & 0.0013 & $3.15(1.55-7.04)$ & 0.0028 \\
\hline Normal & $265(89.2)$ & $285(96.3)$ & 1.00 & & 1.00 & \\
\hline \multicolumn{7}{|l|}{ Uric acid ( $\mu \mathrm{mol} / \mathrm{l})$} \\
\hline High & $10(3.4)$ & $8(2.7)$ & $1.25(0.48-3.22)$ & 0.8117 & $1.18(0.41-3.88)$ & 0.8531 \\
\hline Normal & 287(96.6) & $288(97.3)$ & 1.00 & & 1.00 & \\
\hline \multicolumn{7}{|l|}{ Hb (g/dl) } \\
\hline Anemia & $80(26.9)$ & $57(19.3)$ & $1.55(1.05-2.27)$ & 0.0319 & $1.58(1.11-2.62)$ & 0.0397 \\
\hline Non-anemia & $217(73.1)$ & $239(80.7)$ & 1.00 & & 1.00 & \\
\hline \multicolumn{7}{|l|}{ FBS (mmol/L) } \\
\hline High Normal & $27(9.1)$ & $16(5.4)$ & $1.75(0.92-3.32)$ & 0.1124 & $1.85(0.81-3.85)$ & 0.1068 \\
\hline Normal & $270(90.9)$ & $280(94.6)$ & 1.00 & & 1.00 & \\
\hline \multicolumn{7}{|l|}{ TC (mmol/L) } \\
\hline High & $91(30.6)$ & $76(25.7)$ & $1.27(0.89-1.83)$ & 0.2013 & $1.30(0.94-2.03)$ & 0.2750 \\
\hline Desirable & $206(69.4)$ & $220(74.3)$ & 1.00 & & 1.00 & \\
\hline \multicolumn{7}{|l|}{ TG (mmol/L) } \\
\hline High & $30(10.1)$ & $14(4.7)$ & $2.26(1.17-4.36)$ & 0.0179 & $2.14(1.08-4.79)$ & 0.0206 \\
\hline Normal & $267(89.8)$ & $282(95.3)$ & 1.00 & & 1.00 & \\
\hline \multicolumn{7}{|l|}{ HDL-c (mmol/L) } \\
\hline Low & $18(6.1)$ & $7(2.4)$ & $2.66(1.09-6.47)$ & 0.0390 & $2.57(1.15-7.05)$ & 0.0418 \\
\hline Normal & 279(93.9) & 289(97.6) & 1.00 & & 1.00 & \\
\hline \multicolumn{7}{|l|}{ LDL-c (mmol/L) } \\
\hline High & $54(18.2)$ & $37(12.5)$ & $1.55(0.98-2.44)$ & 0.0679 & $1.38(0.689-2.67)$ & 0.0890 \\
\hline Normal & $243(81.8)$ & $259(87.5)$ & 1.00 & & 1.00 & \\
\hline
\end{tabular}


As shown in Figure 1, there were statistically significantly increased urinary 8-OHdG $(p<0.0001)$ and serum levels of sEng $(p<0.0001)$, sFlt-1 $(p<0.0001), 8$-isoPGF2 $\alpha(p$ $<0.0001)$, 8-OHdG $(p<0.0001)$, sFlt-1: PlGF ratio $(p<0.0001)$, sEng: PlGF ratio $(p$ <0.0001), 8-isoPGF2 $\alpha$ : PIGF ratio $(p<0.0001)$ and 8-OHdG: PIGF ratio $(p<0.0001)$ among pregnant women with SHS compared to those with OHS. Conversely, there were statistically significant low serum levels of PlGF $(p<0.0001)$ and VEGF-A $(p<0.0001)$ among pregnant women with SHS compared to those with OHS. However, the serum levels of TAC were low

357 in pregnant women with SHS compared to those with OHS although there was no statistically significant difference $(\mathrm{p}=0.0860)($ Figure 1). 
A
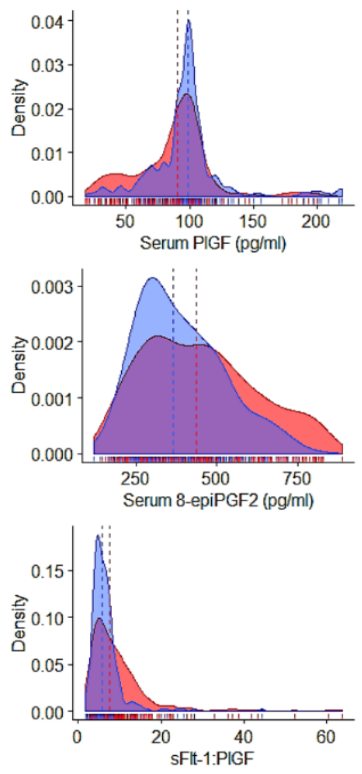

B
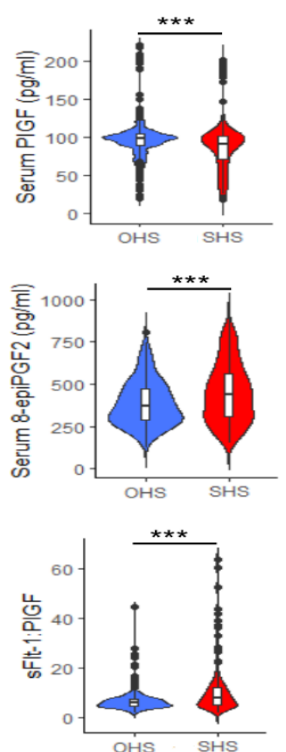

Groups $\vdots$ SHS
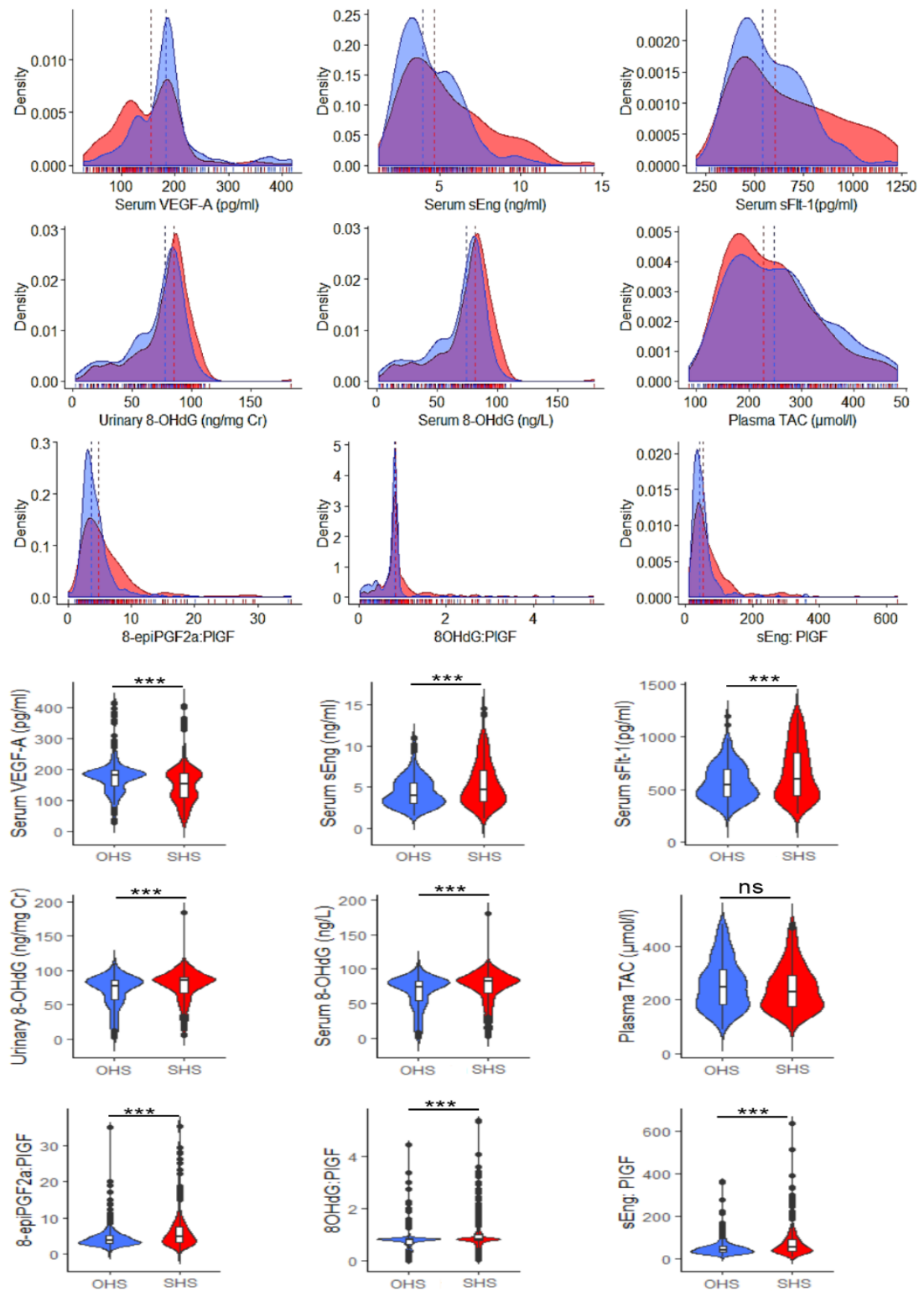

Figure 1. Density (A) and violin (B) plots of individual and combined levels of AGMs and

PIGF: placental growth factor; VEGF-A: Vascular endothelial growth factor-A; sEng: soluble endoglin; sFlt-1: soluble fms-like tyrosine kinase-1; 8-epiPGF2 $\alpha$ : 8-epiprostaglandin F2 alpha; 8-OHdG: 8-hydroxy-2-deoxyguanosine; 

oxidative stress and angiogenic growth mediators

369 As summarised in Table 4, the individual SHS domains such as fatigue, cardiovascular system, immune system and mental health were significant $(p<0.05)$ and negatively associated with PIGF and VEGF-A, but positively associated with sEng, sFlt-1, 8-epiPGF2 $\alpha$, 8-OHdG, sFlt-1/PlGF ratio 8-epiPGF2 $\alpha /$ PlGF ratio, 8-OHdG/PlGF ratio and sEng/PlGF ratio. The SHS-specific domain, 'digestive system' showed the same pattern of results except that there was no significant association with 8-OHdG. TAC was non-significant but negatively associated the individual SHS domains except for the SHS-specific domain, immune system', which showed a significant association. The multivariate model showed that $14.0 \%$ variation in SHS was explained when all the significant independent markers were included in the model (Table 4). 
389 Table 4. Univariate and multivariate linear regression model for individual domain of SHS score in association with obstetric-related

\section{factors, clinical, AGMs and BOS}

\begin{tabular}{|c|c|c|c|c|c|c|c|c|c|c|c|c|}
\hline Parameters $(\mathrm{N}=593)$ & $\begin{array}{l}\text { Fatigue } \\
\mathrm{s} \beta\left(\mathrm{R}^{2}\right)\end{array}$ & $p$-Value & $\begin{array}{l}\text { Cardiovascular } \\
\text { System } \\
\mathrm{s} \beta\left(\mathrm{R}^{2}\right) \\
\end{array}$ & $p$-Value & $\begin{array}{l}\text { Digestive } \\
\text { System } \\
\mathrm{s} \beta\left(\mathrm{R}^{2}\right)\end{array}$ & $p$-Value & $\begin{array}{l}\text { Immune } \\
\text { System } \\
\mathrm{s} \beta\left(\mathrm{R}^{2}\right)\end{array}$ & $p$-Value & $\begin{array}{l}\text { Mental Health } \\
s \beta\left(R^{2}\right)\end{array}$ & $p$-Value & Overall SHS & $p$-Value \\
\hline \multicolumn{13}{|l|}{ Model 1} \\
\hline Age (years) & $-0.007(0.0 \%)$ & 0.8521 & $-0.014(0.0 \%)$ & 0.7369 & $-0.020(0.0 \%)$ & 0.6239 & $-0.003(0.0 \%)$ & 0.9422 & $-0.068(0.5 \%)$ & 0.0982 & $-0.034(0.2 \%)$ & 0.4560 \\
\hline parity & $-0.009(0.0 \%)$ & 0.3593 & $-0.072(0.5 \%)$ & 0.0788 & $-0.015(0.0 \%)$ & 0.7144 & $0.006(0.0 \%)$ & 0.8443 & $-0.028(0.0 \%)$ & 0.4969 & $-0.045(0.2 \%)$ & 0.2771 \\
\hline gravidity & $-0.003(0.0 \%)$ & 0.8113 & $-0.079(0.6 \%)$ & 0.0526 & $-0.026(0.0 \%)$ & 0.5232 & $-0.028(0.0 \%)$ & 0.4949 & $-0.067(0.5 \%)$ & 0.1027 & $-0.051(0.3 \%)$ & 0.2120 \\
\hline Gest. Age (Weeks) & $-0.001(0.0 \%)$ & 0.9683 & $-0.001(0.0 \%)$ & 0.9879 & $-0.022(0.0 \%)$ & 0.5896 & $0.014(0.0 \%)$ & 0.7394 & $-0.007(0.0 \%)$ & 0.8623 & $-0.005(0.1 \%)$ & 0.9040 \\
\hline $\mathrm{SBP}(\mathrm{mmHg})$ & $0.077(0.6 \%)$ & 0.0616 & $0.090(0.8 \%)$ & 0.0281 & $0.058(0.3 \%)$ & 0.1579 & $0.130(1.7 \%)$ & 0.0015 & $0.148(2.2 \%)$ & 0.0003 & $0.144(2.1 \%)$ & 0.0004 \\
\hline $\mathrm{DBP}(\mathrm{mmHg})$ & $-0.009(0.0 \%)$ & 0.8285 & $0.036(0.1 \%)$ & 0.3814 & $-0.001(0.0 \%)$ & 0.9781 & $0.067(0.5 \%)$ & 0.1042 & $0.183(3.3 \%)$ & $<0.0001$ & $0.086(0.6 \%)$ & 0.0375 \\
\hline Gestational BMI & $-0.004(0.0 \%)$ & 0.8980 & $0.084(0.7 \%)$ & 0.0400 & $0.060(0.4 \%)$ & 0.1442 & $0.017(0.0 \%)$ & 0.6834 & $0.038(0.1 \%)$ & 0.3499 & $0.038(0.1 \%)$ & 0.3608 \\
\hline Pre-gestation BMI & $-0.029(0.1 \%)$ & 0.4745 & $0.186(0.5 \%)$ & 0.0867 & $0.046(0.2 \%)$ & 0.2673 & $0.001(0.0 \%)$ & 0.9685 & $0.022(0.0 \%)$ & 0.5990 & $0.010(0.0 \%)$ & 0.8064 \\
\hline PIGF (pg/mL) & $-0.123(1.5 \%)$ & 0.0028 & $-0.114(1.3 \%)$ & 0.0054 & $-0.150(2.3 \%)$ & 0.0002 & $-0.167(2.8 \%)$ & $<0.0001$ & $-0.175(3.1 \%)$ & $<0.0001$ & $-0.207(4.3 \%)$ & $<0.0001$ \\
\hline VEGF-A $(p g / m L)$ & $-0.142(2.0 \%)$ & 0.0005 & $-0.143(2.0 \%)$ & 0.0005 & $-0.140(2.0 \%)$ & 0.0006 & $-0.164(2.7 \%)$ & $<0.0001$ & $-0.202(4.1 \%)$ & $<0.0001$ & $-0.230(5.3 \%)$ & $<0.0001$ \\
\hline sEng (ng/mL) & $0.186(3.5 \%)$ & $<0.0001$ & $0.101(1.0 \%)$ & 0.0137 & $0.087(0.8 \%)$ & $\mathbf{0 . 0 3 3 3}$ & $0.097(1.0 \%)$ & 0.0177 & $0.155(2.4 \%)$ & 0.0002 & $0.212(4.5 \%)$ & $<0.0001$ \\
\hline sFlt-1 (pg/ml) & $0.182(3.3 \%)$ & $<0.0001$ & $0.155(2.4 \%)$ & 0.0001 & $0.162(2.6 \%)$ & $<0.0001$ & $0.209(4.4 \%)$ & $<0.0001$ & $0.208(4.3 \%)$ & $<0.0001$ & $0.270(7.3 \%)$ & $<0.0001$ \\
\hline 8-epiPGF $2 \alpha(\mathrm{pg} / \mathrm{ml})$ & $0.139(1.9 \%)$ & 0.0007 & $0.138(1.9 \%)$ & 0.0008 & $0.136(1.8 \%)$ & 0.0009 & $0.148(2.2 \%)$ & 0.0003 & $0.206(4.3 \%)$ & $<0.0001$ & $0.225(5.1 \%)$ & $<0.0001$ \\
\hline 8-OHdG(ng/mgCr) & $0.119(1.4 \%)$ & 0.0037 & $0.110(1.2 \%)$ & 0.0073 & $0.058(0.3 \%)$ & 0.1683 & $0.128(1.6 \%)$ & 0.0019 & $0.158(2.5 \%)$ & 0.0001 & $0.175(3.1 \%)$ & $<0.0001$ \\
\hline U8-OHdG(ng/ml) & $0.125(1.6 \%)$ & 0.0023 & $0.101(1.0 \%)$ & 0.0134 & $0.069(0.5 \%)$ & 0.0956 & $0.140(2.0 \%)$ & 0.0006 & $0.151(2.3 \%)$ & 0.0002 & $0.178(3.2 \%)$ & $<0.0001$ \\
\hline $\mathrm{TAC}(\mu \mathrm{mol} / \mathrm{L})$ & $-0.062(0.4 \%)$ & 0.1301 & $-0.002(0.0 \%)$ & 0.9560 & $-0.003(0.0 \%)$ & 0.9345 & $-0.100(1.0 \%)$ & 0.0178 & $-0.045(0.2 \%)$ & 0.2775 & $-0.072(0.5 \%)$ & 0.0883 \\
\hline sFlt-1: PIGF ratio & $0.177(3.2 \%)$ & $<0.0001$ & $0.205(4.2 \%)$ & $<0.0001$ & $0.167(2.8 \%)$ & $<0.0001$ & $0.248(6.1 \%)$ & $<0.0001$ & $0.233(5.4 \%)$ & $<0.0001$ & $0.292(8.5 \%)$ & $<0.0001$ \\
\hline sEng: PIGF ratio & $0.160(2.6 \%)$ & $<0.0001$ & $0.149(2.2 \%)$ & 0.0003 & $0.140(2.0 \%)$ & 0.0006 & $0.194(3.8 \%)$ & $<0.0001$ & $0.193(3.7 \%)$ & $<0.0001$ & $0.244(6.0 \%)$ & $<0.0001$ \\
\hline 8-epiPGF2 $\alpha$ : PIGF & $0.146(2.1 \%)$ & 0.0004 & $0.187(3.5 \%)$ & $<0.0001$ & $0.163(2.7 \%)$ & $<0.0001$ & $0.223(5.0 \%)$ & $<0.0001$ & $0.223(5.0 \%)$ & $<0.0001$ & $0.262(6.9 \%)$ & $<0.0001$ \\
\hline 8-OHdG: PIGF & $0.141(2.0 \%)$ & 0.0006 & $0.167(2.8 \%)$ & $<0.0001$ & $0.159(2.5 \%)$ & $<0.0001$ & $0.219(4.8 \%)$ & $<0.0001$ & $0.211(4.4 \%)$ & $<0.0001$ & $0.250(6.3 \%)$ & $<0.0001$ \\
\hline \multicolumn{13}{|l|}{ Model 2} \\
\hline $\mathrm{R}^{2}$ & $7.2 \%$ & & $5.1 \%$ & & $5.0 \%$ & & $10.7 \%$ & & $11.4 \%$ & & $14.0 \%$ & \\
\hline Adjusted $\mathrm{R}^{2}$ & $5.5 \%$ & & $2.9 \%$ & & $3.5 \%$ & & $8.8 \%$ & & $9.4 \%$ & & $12.2 \%$ & \\
\hline \multicolumn{13}{|l|}{ Constant } \\
\hline $\mathrm{p}$-value & $<0.0001$ & & 0.0042 & & 0.0004 & & $<0.0001$ & & $<0.0001$ & & $<0.0001$ & \\
\hline
\end{tabular}

$s \beta\left(\mathrm{R}^{2}\right)$ : Standardised regression coefficient (Coefficient of determination); SBP: systolic blood pressure; DBP: diastolic blood pressure, PIGF: placental growth factor; VEGF-A: Vascular endothelial growth factor-A; sEng: soluble endoglin; sFlt-1: soluble fms-like tyrosine kinase-1; 8-epiPGF2 $\alpha$ : 8-epiprostaglandin F2 alpha; 8-OHdG: 8-hydroxy-2-deoxyguanosine; TAC: Total antioxidant capacity. Univariate (Model 1); Multivariate (Model 2): included all significant parameters in the model 

There was a significantly negative association between SHS and serum PlGF ${ }_{s} \beta=-$ $\left.0.207 ; \mathrm{R}^{2}=4.3 \% ; p<0.0001\right)$ and VEGF-A $\left({ }_{\mathrm{s}} \beta=-0.230 ; \mathrm{R}^{2}=5.3 \% ; p<0.0001\right)$ but a significantly positive association with $\mathrm{sEng}\left({ }_{\mathrm{s}} \beta=0.212 ; \mathrm{R}^{2}=4.5 \% ; p<0.0001\right)$, and sFlt-1 ${ }_{\mathrm{s}} \beta$ $\left.=0.270 ; \mathrm{R}^{2}=7.3 \% ; p<0.0001\right)$ There was a significantly positive association between SHS and serum 8-epiPGF2 $\alpha\left({ }_{s} \beta=0.225 ; \mathrm{R}^{2}=5.1 \% ; p<0.0001\right)$, serum 8 -OHdG ${ }_{(s} \beta=0.175 ; \mathrm{R}^{2}=$ $3.1 \% ; p<0.0001)$, and urinary 8 - $\mathrm{OHdG}\left({ }_{\mathrm{s}} \beta=0.179 ; \mathrm{R}^{2}=3.2 \% ; p<0.0001\right)$ but a negative relationship between SHS and TAC $\left({ }_{s} \beta=-0.720 ; \mathrm{R}^{2}=0.5 \% ; p=0.0883\right.$ ) (Figure 2). 


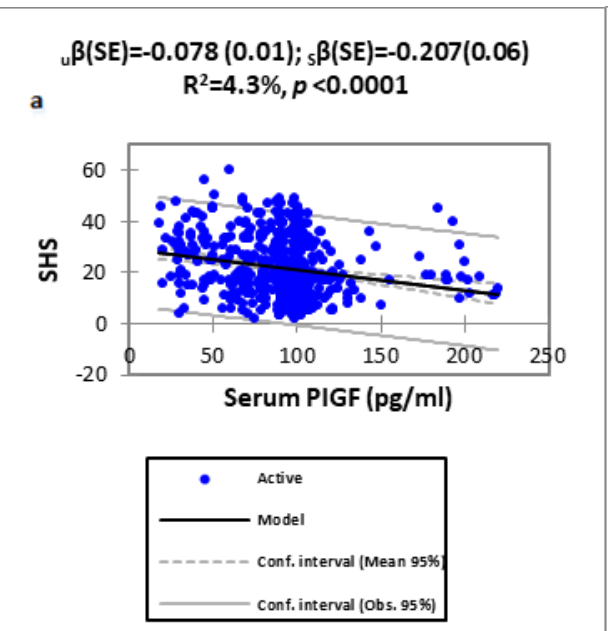

${ }_{u} \beta(S E)=1.033(0.18) ;{ }_{s} \beta(S E)=0.212(0.04$ $R^{2}=4.5 \%, p<0.0001$
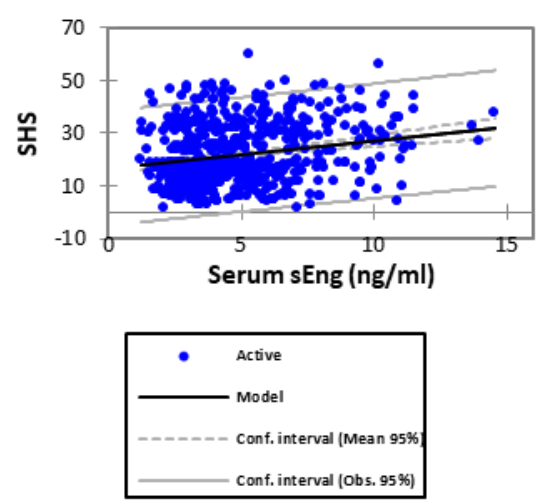

${ }_{u} \beta(S E)=-0.041(0.00) ;{ }_{s} \beta(S E)=-0.23(0.04)$

b
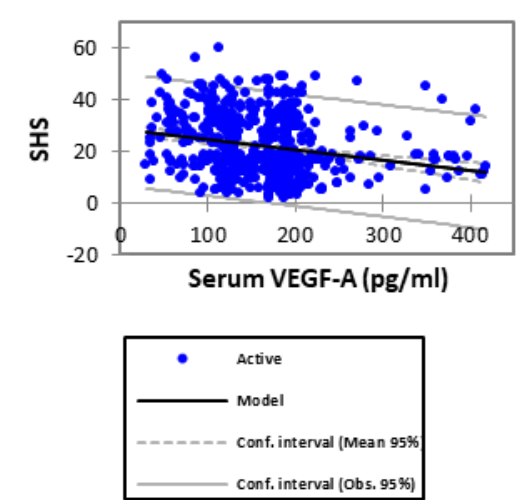

d $\quad u(S E)=0.014(0.00) ;{ }_{5} \beta(S E)=0.270(0.04)$ $R^{2}=7.3 \%, p<0.0001$
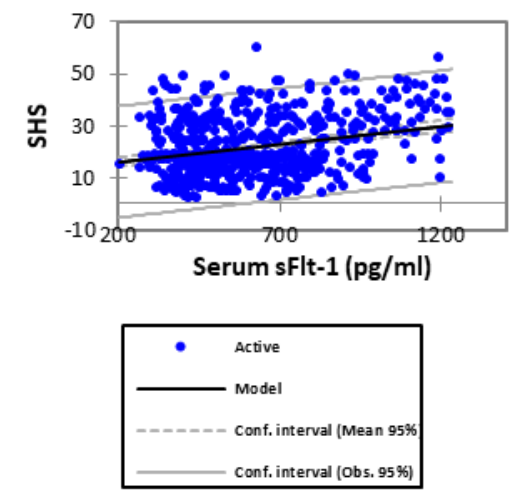

${ }_{u} \beta(S E)=0.016(0.00) ;{ }_{5} \beta(S E)=0.226(0.04)$ $R^{2}=5.1 \%, p<0.0001$

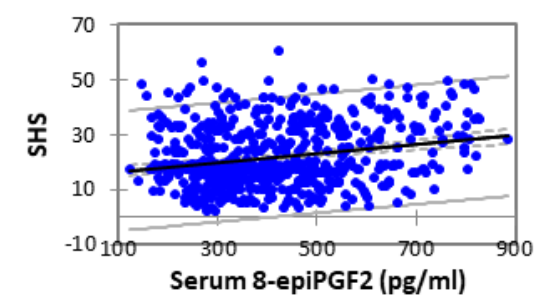

Serum 8-epiPGF2 $(\mathrm{pg} / \mathrm{ml})$

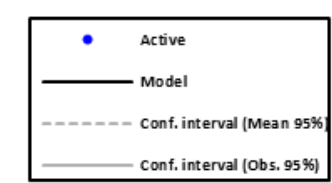

g $\beta(S E)=0.086(0.00) ; \beta(S E)=0.179(0.04)$ $R^{2}=3.2 \%, p<0.0001$
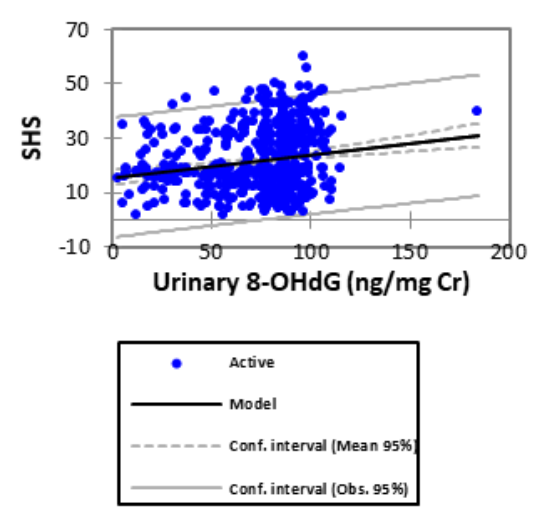

${ }_{u} \beta(S E)=0.085(0.01) ; \beta(S E)=0.175(0.04)$ $R^{2}=3.1 \%, p<0.0001$

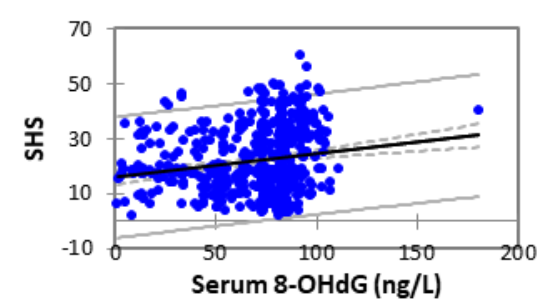

Serum 8-OHdG (ng/L)

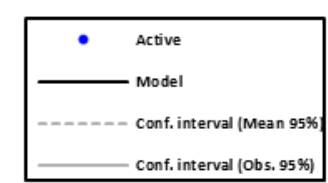

${ }_{u} \beta(\mathrm{SE})=-0.009(0.00) ; \beta(\mathrm{SE})=-0.070(0.04)$ $\mathrm{R}^{2}=0.5 \%, p=0.0883$
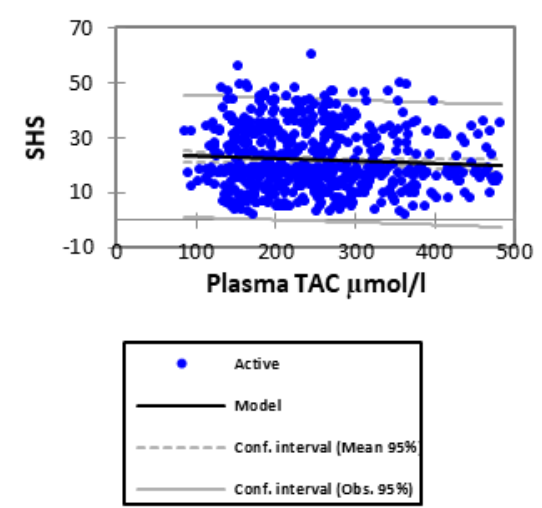

\section{Figure 2. Linear regression model of SHS score in association with levels of AGMs and BOS among NTN-PW}

u $\beta$ : unstandardised co-efficient; s $\beta$ : standardised co-efficient; SE: standard error; $\mathrm{R}^{2}$ : coefficient of determination. Significant negative association between SHS and serum PIGF ( $\beta=-0.207$; $p<0.0001$ ) (Figure 2a); and VEGF-A ( $\beta=-0.230 ; p<0.0001$ ) (Figure 2b). Significant positive association between SHS and $\mathrm{sEng}$ ( $\mathrm{s} \beta=0.212 ; p<0.0001)$ (Figure 2c); sFlt-1 (s $\beta=0.270 ; p$ $<0.0001$ ) (Figure 2d); serum 8-epiPGF2 $\alpha$ (s $\beta=0.225 ; p<0.0001$ ) (Figure 2e); serum 8-OHdG (s $\beta=0.175 ; p<0.0001$ ) (Figure 2f) and urinary 8-OHdG (s $\beta=0.179 ; p<0.0001$ ) (Figure 2g).

$410<0.0001)($ Figure 2d); serum 8-epiPGF2 $\alpha(\mathrm{s} \beta=0.225 ; p<0.0001)$ (Figure 2e); serum 8-OHdG $(\mathrm{s} \beta=0.175 ; p$
$411 \quad$ Non-significant negative relationship between SHS and TAC $\left(\mathrm{s} \beta=-0.720 ; \mathrm{R}^{2}=0.5 \% ; p=0.0883\right.$ (Figure $2 \mathbf{h}$ ) 
412 Relationship between the overall SHS score and combined biomarkers of oxidative stress and angiogenic growth mediators

As shown in Figure 3, there was a significantly positive relationship between SHS 415 and sFlt-1: PIGF ratio $\left({ }_{s} \beta=0.292 ; \mathrm{R}^{2}=8.5 \% ; p<0.0001\right), 8$-epiPGF2 $\alpha$ : PlGF ratio $\left({ }_{s} \beta=0.262\right.$; $\left.416 \mathrm{R}^{2}=6.9 \% ; p<0.0001\right), 8$-OHdG: PlGF ratio $\left(\mathrm{s} \beta=0.250 ; \mathrm{R}^{2}=6.3 \% ; p<0.0001\right)$, sEng: PlGF 417 ratio $\left.\left({ }_{s} \beta=0.244 ; \mathrm{R}^{2}=6.0 \% ; p<0.0001\right), \mathrm{SBP}{ }_{\mathrm{s}} \beta=0.144 ; \mathrm{R}^{2}=2.1 \% ; p=0.0004\right)$ and $\mathrm{DBP}\left({ }_{s} \beta\right.$ $\left.418=0.086 ; \mathrm{R}^{2}=0.7 \% ; p=0.0375\right)($ Figure 3). 


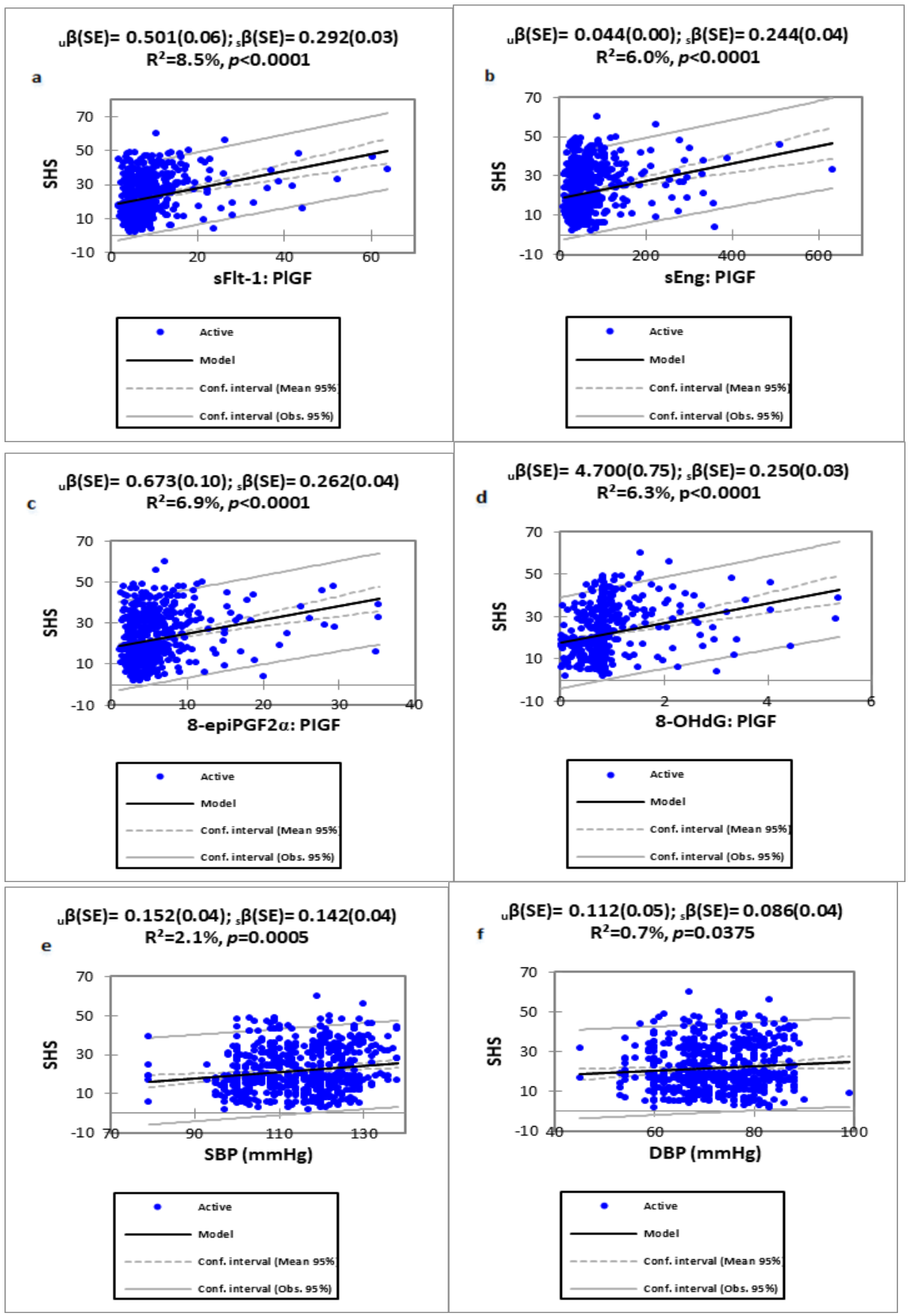

\section{Figure 3. Linear regression model of SHS score in association with ratios of AGMs and} BOS and BP among NTN-PW

$\mathrm{u} \beta$ : unstandardised co-efficient; s $\beta$ : standardised co-efficient; SE: standard error; $\mathrm{R}^{2}$ : coefficient of determination. Significant positive association between SHS and sFlt-1: PlGF ratio ( $\mathrm{s} \beta=0.292 ; p<0.0001$ ) (Figure 3a); sEng: PlGF ratio ( $\mathrm{s} \beta=0.244 ; p$ $<0.0001$ ) (Figure 3b), 8-epiPGF2 $\alpha$ : PlGF ratio ( $\beta=0.262 ; p<0.0001$ ) (Figure 3c), 8-OHdG: PlGF ratio ( $s \beta=0.250 ; p$ $<0.0001$ ) (Figure 3d), $\mathrm{SBP}(\mathrm{s} \beta=0.144 ; p=0.0004)$ (Figure 3e) and DBP $(\mathrm{s} \beta=0.086 ; p=0.0375)$ (Figure 3f). 
As shown in Table 5 the $1^{\text {st }}$ quartiles for serum PIGF [aOR=2.79; 95\% CI (1.43 to $3.28) ; p=0.0002]$ and VEGF-A [aOR $=5.35 ; 95 \% \mathrm{CI}(2.85$ to 10.01$) ; p<0.0001]$, the $2^{\text {nd }}$ quartile for PIGF [aOR $=2.48 ; 95 \% \mathrm{CI}(1.28$ to 5.29$)] ; p=0.0154)$ and the $4^{\text {th }}$ quartiles for sEng $[\mathrm{aOR}=4.31 ; 95 \% \mathrm{CI}(2.37$ to 7.81$) ; p<0.0001]$, sFlt-1[aOR $=1.84 ; 95 \% \mathrm{CI}(1.15$ to $2.83) ; p=0.0013], 8$-epiPGF $\alpha$ [aOR $=2.23 ; 95 \%$ CI $(1.41$ to 3.46$) ; p=0.0001]$, serum 8 $\mathrm{OHdG}[\mathrm{aOR}=1.90 ; 95 \% \mathrm{CI}(1.28$ to 2.83$) ; p=0.0018]$ and urinary $8-\mathrm{OHdG}[\mathrm{aOR}=1.95$; 95\% CI (1.30 to 2.90); $p=0.0004$ ] were independently associated with SHS with only few variations in the odds ratios after adjusting for confounding factors (Table 5).

440

441

442

443

444

445

446

447

448

449

450 


\begin{tabular}{|c|c|c|c|c|c|c|}
\hline Parameters & $\begin{array}{c}\text { SHS } \\
(\mathrm{N}=297)\end{array}$ & $\begin{array}{c}\text { OHS } \\
(\mathrm{N}=296)\end{array}$ & $\begin{array}{c}\text { Crudes odds ratio } \\
(95 \% \mathrm{CI})\end{array}$ & $p$-value & $\begin{array}{c}\text { adjusted odds ratio } \\
(95 \% \mathrm{CI})\end{array}$ & $p$-value \\
\hline \multicolumn{7}{|c|}{ Serum PIGF (pg/ml) } \\
\hline Q1 (<80.10) & $91(30.6)$ & $56(18.9)$ & $2.12(1.39$ to 3.24$)$ & 0.0005 & $2.79(1.43$ to 3.28$)$ & 0.0002 \\
\hline Q2 (80.10-89.10) & $27(9.1)$ & $14(4.7)$ & $2.52(1.26$ to 5.05$)$ & 0.0104 & $2.48(1.28$ to 5.29$)$ & 0.0154 \\
\hline Q3 (89.11-99.10) & $78(26.3)$ & $94(31.8)$ & $1.08(0.73$ to 1.61$)$ & 0.7615 & $1.13(0.81$ to 1.77$)$ & 0.4382 \\
\hline Q4 (>99.11) & $101(34.0)$ & $132(44.6)$ & 1.00 & & 1.00 & \\
\hline \multicolumn{7}{|c|}{ Serum VEGF-A (pg/ml) } \\
\hline Q1 (<124.4) & $108(36.4)$ & $40(13.5)$ & $5.31(2.98$ to 9.43$)$ & $<0.0001$ & $5.35(2.85$ to 10.01$)$ & $<0.0001$ \\
\hline Q2 (124.4-163.4) & $45(15.1)$ & $50(16.9)$ & $1.76(0.96$ to 3.22$)$ & 0.0704 & $1.63(0.91$ to 3.25$)$ & 0.0816 \\
\hline Q3 (163.5-203.4) & $115(38.7)$ & $149(50.3)$ & $1.51(0.91$ to 2.52$)$ & 0.1299 & $1.39(0.60$ to 2.81$)$ & 0.3014 \\
\hline Q4 (>203.5) & $29(9.8)$ & $57(19.3)$ & 1.00 & & 1.00 & \\
\hline \multicolumn{7}{|c|}{ Serum sEng (ng/ml) } \\
\hline $\mathrm{Q} 1(<3.194)$ & $65(21.9)$ & $83(28.0)$ & 1.00 & & 1.00 & \\
\hline Q2 (3.194-5.194) & $106(35.7)$ & $114(23.6)$ & $1.19(0.78$ to 1.81$)$ & 0.4563 & $1.15(0.76$ to 1.82$)$ & 0.5035 \\
\hline Q3 (5.195-7.195) & $59(19.9)$ & $79(26.7)$ & $0.95(0.59$ to 1.52$)$ & 0.9051 & $1.07(0.64$ to 1.69$)$ & 0.9713 \\
\hline Q4 (>7.196) & $67(22.6)$ & $20(6.8)$ & $4.28(2.35$ to 7.76$)$ & $<0.0001$ & $4.31(2.37$ to 7.81$)$ & $<0.0001$ \\
\hline \multicolumn{7}{|c|}{ Serum sFlt-1 (pg/ml) } \\
\hline Q1 (<441.3) & $69(23.2)$ & $78(26.4)$ & 1.00 & & 1.00 & \\
\hline Q2 (441.3-561.3) & $62(20.9)$ & $83(28.0)$ & $0.84(0.53$ to 1.34$)$ & 0.4827 & $0.88(0.51$ to 1.40$)$ & 0.3016 \\
\hline Q3 (561.4-681.4) & $40(13.5)$ & $57(19.3)$ & $0.79(0.47$ to 1.33$)$ & 0.4305 & $0.80(0.48$ to 1.35$)$ & 0.3580 \\
\hline Q4 (>681.5) & $126(42.4)$ & $78(26.4)$ & $1.83(1.18$ to 2.81$)$ & 0.0066 & $1.84(1.15$ to 2.83$)$ & 0.0013 \\
\hline \multicolumn{7}{|c|}{ Serum 8-epiPGF2a (pg/ml) } \\
\hline Q1 (<295.0) & $64(21.5)$ & $84(28.4)$ & 1.00 & & 1.00 & \\
\hline Q2( 295.0-394.0) & $64(21.5)$ & $81(27.4)$ & $1.04(0.65$ to 1.64$)$ & 0.9066 & $1.13(0.58$ to 1.66$)$ & 0.9801 \\
\hline Q3 (395.0-494.0) & $60(20.2)$ & $66(22.3)$ & $1.19(0.74$ to 1.92$)$ & 0.5427 & $1.16(0.70$ to 1.97$)$ & 0.4911 \\
\hline Q4 (>495.0) & $109(36.7)$ & $65(21.9)$ & $2.20(1.40$ to 3.44$)$ & 0.0005 & $2.23(1.41$ to 3.46$)$ & 0.0001 \\
\hline \multicolumn{7}{|c|}{ Serum 8-OHdG (ng/L) } \\
\hline $\mathrm{Q} 1(<61.40)$ & $61(20.5)$ & $87(29.4)$ & 1.00 & & 1.00 & \\
\hline Q2(61.40-71.40) & $21(7.1)$ & $24(8.1)$ & $1.24(0.63$ to 2.44$)$ & 0.6059 & $1.21(0.67$ to 2.53$)$ & 0.5473 \\
\hline Q3(71.50-81.50) & $51(17.2)$ & $61(20.6)$ & $1.19(0.73$ to 1.96$)$ & 0.528 & $1.15(0.69$ to 1.98$)$ & 0.6014 \\
\hline $\mathrm{Q} 4(>81.60)$ & $164(55.2)$ & $124(41.9)$ & $1.89(1.26$ to 2.82$)$ & 0.0024 & $1.90(1.28$ to 2.83$)$ & 0.0018 \\
\hline \multicolumn{7}{|c|}{ Urinary 8-OHdG (ng/mg Cr) } \\
\hline Q1 (<59.95) & $61(20.5)$ & $87(29.4)$ & 1.00 & & 1.00 & \\
\hline Q2 (59.95-69.95) & $21(7.1)$ & $21(7.1)$ & $1.43(0.72$ to 2.83$)$ & 0.3779 & $1.42(0.71$ to 2.85$)$ & 0.3506 \\
\hline Q3 (69.96-79.96) & $52(17.5)$ & $67(22.6)$ & $1.11(0.68$ to 1.80$)$ & 0.7097 & $1.18(0.64$ to 1.81$)$ & 0.6937 \\
\hline $\mathrm{Q} 4(>79.97)$ & $163(54.9)$ & $121(40.9)$ & $1.92(1.28$ to 2.87$)$ & 0.0016 & $1.95(1.30$ to 2.90$)$ & 0.0004 \\
\hline \multicolumn{7}{|c|}{ Plasma TAC $(\mu \mathrm{mol} / \mathrm{L})$} \\
\hline Q1 (<178.9) & $78(26.3)$ & $70(23.6)$ & $1.20(0.82$ to 1.77$)$ & 0.3749 & $1.48(0.99$ to 1.76$)$ & 0.0504 \\
\hline Q2 (178.9-198.9) & $37(12.5)$ & $28(9.5)$ & $1.43(0.84$ to 2.44$)$ & 0.2228 & $1.41(0.81$ to 1.71$)$ & 0.3001 \\
\hline Q3 (199.9-219.9) & $21(7.1)$ & $24(8.1)$ & $0.95(0.51$ to 1.76$)$ & 0.8753 & $0.98(0.51$ to 1.78$)$ & 0.7937 \\
\hline Q4 (>220.9) & $161(54.2)$ & $174(58.8)$ & 1.00 & & 1.00 & \\
\hline
\end{tabular}

Values are presented as frequency (proportion); odds ratio (95\% confidence intervals). 1.00 (reference category). Q: quartile. PIGF: placental growth factor; VEGF-A: Vascular endothelial growth factor-A; sEng: soluble endoglin; sFlt-1: soluble fmslike tyrosine kinase-1; 8-epiPGF2 $\alpha$ : 8-epiprostaglandin F2 alpha; 8-OHdG: 8-hydroxy-2-deoxyguanosine; TAC: Total antioxidant capacity. Covariate of adjusted model include maternal age, parity, gravidity, high BP, family history of hypertension, history of spontaneous abortion, pre-gestational BMI, high TG, AST, LDH, creatinine, and low Hb, low HDL, low $\mathrm{Mg}$ and low Ca. 
Discussion

Using the subjective and non-invasive SHSQ-25, we stratified the health status of NTN-PW into SHS and optimal health status (OHS), compared the levels of OS and AGMs in these SHS and OHS groups and further tested the association between SHS and these biomarkers by performing a linear regression and multivariate logistic regression model. Overall, our novel findings indicated that the higher the SHS score, the more deranged the levels of BOS and AGMs, and this was further confirmed by a significant independent association between them after adjusting for confounding factors: maternal age, parity, gravidity, high BP, family history of hypertension, history of spontaneous abortion, pre-gestational BMI, high TG, AST, $\mathrm{LDH}$, creatinine, and low $\mathrm{Hb}, \mathrm{HDL}, \mathrm{Mg}$ and $\mathrm{Ca}$.

Particularly, NTN-PW with SHS had significantly increased OS biomarkers as depicted by increased levels of the pro-oxidants (8-OHdG, 8-epiPGF2 $\alpha$ ) and a fairly reduced antioxidant (TAC) (Figure 1). This was confirmed by a significant positive association between SHS and 8-OHdG and 8-epiPGF2 $\alpha$ but a negative association with TAC, which means that SHS increases with increasing pro-oxidant activity and a reduced anti-oxidant system. This finding might be indicative of increased oxidative DNA damage, endogenous oxidative stress and a compromised anti-oxidant system [8]. Previous studies have also explained that an incomplete maternal vascular artery remodeling results in placental hypoxia/ischaemia, which eventually leads to OS [6,8]. Cardiovascular risk factors including dyslipidaemia and hypertension have also been linked to OS $[33,34]$. Hormonal imbalances are commonly associated with pregnancy and it is reported to contribute to increased phospholipid accumulation [12]. In the present study, cardiovascular indicators such as high triglyceride, low HDL-c and high BP were found to be independent risk factors for SHS (Table 3). The association was confirmed by positive correlation between the SHS-specific domain, 'cardiovascular system' and unbalanced BOS (Table 4). This finding is consistent 
with a cross-sectional study conducted in a Chinese population which observed an association between SHS and ideal cardiovascular health metrics [26]. The observed increased OS among SHS individuals may further be explained by these cardiovascular risk factors associated with SHS. Hormonal imbalances are commonly associated with pregnancy and it is reported to contribute to increased phospholipid accumulation [12]. Increased lipids at sites where ROS are formed can result in endogenous lipid peroxidation [12]. Hence, the observed increased OS among SHS NTN-PW may possibly be due to ROS-induced lipid peroxidation [12]. In order to understand the strength of the association between OS and SHS, we adjusted for the significant haematobiochemical, clinical and obstetrics factors associated with SHS. Interestingly, the association between SHS and OS biomarkers still remained significant with slight variations in the odds ratios, indicating that the association is independent of confounding factors. Particularly as shown in Table 5, the fourth quartile for serum 8-epiPGF2 $\alpha$, serum 8-OHdG and urinary 8-OHdG showed a 2.23-fold, 1.90-fold and 1.95-fold increased adjusted odds of SHS compared to the first quartile levels. This finding makes SHS an independent risk factor for OS. We therefore hypothesize that SHS is associated with increased OS and poor maternal vascular remodeling compared to pregnant women with optimal health. This would inform clinicians the need for a combined antioxidant supplement and pro-angiogenic molecules. Evaluation of SHS criterion can create an opportunity for predictive, preventive and personalised medicine.

Increased OS during normotensive pregnancy, although not well understood may also be attributed to several mechanisms. For instance, maternal anaemia is reported as one major risk factor that contributes significantly to increase OS [3]. In the present study, the significant association between maternal low haemoglobin levels and SHS may be a contributing factor for the observed increased OS among SHS compared to optimal health NTN-PW; however, the relationship between OS and SHS was independent of anaemia. 
513 Also, psychosocial stress which is a health complaint commonly among pregnant has been

514 linked with OS [4]. A cross-sectional study among normal pregnant women reported that an

515 increased OS may be associated with maternal psychosocial stress [4]. Another cross-

516 sectional study among an adult Chinese population also found a significant relationship

517 between SHS and psychosocial stress [28]. In the present study, 'fatigue', which is an index 518 of psychosocial stress, and also one of the SHS domains was associated with increased OS 519 and a compromised antioxidant system (Table 4). The observed OS among SHS participants may be somewhat due to its association with the SHS domain, 'fatigue'. Increased OS has also been associated with dietary magnesium $(\mathrm{Mg})$ and calcium $(\mathrm{Ca})$ deficiencies $[35,36]$, even though the associations are still debateable. In the present study, low $\mathrm{Mg}$ and $\mathrm{Ca}$ levels were significantly associated with SHS. Decreased $\mathrm{Mg}$ and Ca levels stimulate increased release of catecholamine, which can further increase the production and formation of ROS and result into OS [37]. In addition, Mg deficiency may induce ROS formation and lead to OS via activation of the renin-angiotensin-aldosterone system (RAAS) [37]. Mg deficiency is also reported as an early marker of endothelial dysfunction, which is also a complication of OS [35,36]. The relationship between SHS and increased OS observed in the present study may partly be due to the hypomagnesaemia and hypocalcaemia observed among SHS participants. Thus, early identification of SHS along with low Mg and Ca levels can inform clinicians of the pregnant women who stand the risk of increased OS, thus allowing the need to administer magnesium and calcium supplementations to prevent OS and possible adverse perinatal outcome.

Another major novel finding in the present study was the significantly reduced PlGF and VEGF-A levels and a correspondingly increased sFlt-1 and sEng among SHS compared to optimal health NTN-PW (Figure 1). This finding signifies that SHS NTN-PW may have suffered an overexpression of anti-angiogenic growth mediators which has in turn interfered 
with the pro-angiogenic function. The imbalance in AGMs observed in the present study was further confirmed by a significantly negative association of SHS with PIGF and VEGF-A, but a positive association with sFlt-1 and sEng (Figure 2). These imbalances could possibly be explained as a local placental ischaemia originating from incomplete maternal vascular remodeling which has increased systemic OS culminating in a shift in function in favour of sFlt-1 [7]. Increased OS is reported to stimulate the antagonistic activity of sFlt-1, which in turn neutralises the function of VEGF-A and PIGF [7,8]. The increased OS and unbalanced AGMs among SHS NTN-PW is a clear indication of a compromised immune health, as both factors play important role in the immune response of pregnancy. Our present study found an association between the SHS-specific domain, 'immune system', and increased OS and imbalance in pro-and anti-AGMs (Table 4).

Also, the reduced PIGF and VEGF-A concentration and increased anti-AGMs (sFlt-1 and sEng) observed among SHS NTN-PW in the present study can be linked to an event of endothelial dysfunction. While VEGF-A is an essential factor for regulating the endothelium, sEng may interfere with endothelial control by inhibiting the function of TGF $\beta 1$, which plays a central role in nitric oxide (NO) production and vasodilation [8]. The relationship between SHS and an imbalance in AGMs may be explained by the increased OS observed among SHS participants. A cross-sectional study found a significant association between SHS and endothelial dysfunction in an adult Russian population [24]. Endothelial dysfunction, although mostly associated with preeclamptic pregnancies can also be associated with uncomplicated pregnancies due to physiological adaptations [38]. The first quartile for VEGF-A and PIGF and the fourth quartile for sFlt-1 and sEng were independently associated with SHS. The first quartile for VEGF-A and PIGF were 5.35 and 2.79 times, and sFlt-1 and sEng were 1.84 and 4.31 times increased adjusted odds of SHS, respectively (Table 5). This supports our findings that SHS is associated with an imbalance in AGMs in pregnancy and 
thus, incorporating SHSQ-25 as a tool in early antenatal health screening can be used as a risk stratification for abnormal maternal vascular remodeling and placental angiogenesis. This can create an opportunity for clinicians to detect early and administer appropriate medicinal intervention such as angiogenic molecules to SHS pregnant women to prevent likely adverse pregnancy outcomes.

Previous studies have reported that an algorithm of markers explain and predict better the physiological variation in a condition compared using the individual markers $[8,39]$. In the present study, we created a novel combined OS/AGMs ratio: 8-epiPGF2/P1GF and 8OHdG/PIGF in addition to the previously known ratios: sFlt-1/PIGF and sEng/PIGF. There were significantly increased levels of sFlt-1/PlGF, 8-epiPGF2/PlGF, 8-OHdG/PIGF and sEng/PIGF ratios among SHS compared to OHS NTN-PW (Figure 1). Based on this finding, we performed a linear regression model and found a significantly positive association between these ratios and SHS. A higher percentage coefficient of variation in SHS was explained by these combined markers compared to using the individual markers (Figure 3). Increased levels of these combined markers among SHS NTN-PW support our present study findings that an imbalance in AGMs and increased OS are associated with SHS. Hence, we hypothesize that these combined panel markers can be used as a potential diagnostic tool for OS-induced abnormal placental angiogenesis and are likely to be useful generic markers of adverse pregnancy outcomes. The observed association signifies that SHS, oxidative stress and placental angiogenesis may exhibit a synergistic physiological function.

While the findings in the present study are novel, there were some limitations. Firstly, because the present study is a cross-sectional hospital-based study, our results cannot be generalised for the entire population. Nevertheless, this study is the baseline of an ongoing prospective GHOACS. Aside from these limitations, there were some strengths to highlight. This is the first cross-sectional study which sought to ascertain if 
SHS is associated with increased OS and unbalanced AGMs among normotensive pregnant women in a Ghanaian. Another strength of the present study finding was that the association remained significant after adjusting for confounding factors, indicating that SHS is an independent risk factor of increased OS and unbalanced AGMs.

\section{Conclusion}

In summary, increase oxidative stress and imbalances in pro and-anti-angiogenic growth mediators are independently associated with SHS. This was supported by an association of OS and AGMs with the individual SHS-specific domains. SHSQ-25 evaluation, which is a subjective non-invasive assessment for SHS can be used to identify increased OS and poor maternal vascular remodeling and thus inform clinicians of the need for antioxidant supplementation. Evaluation of SHSQ-25 may be an effective and timeefficient tool that can augment other point-of-care testing especially in resource-limited facility in sub-Saharan African to improve poor health among normotensive pregnant women who suffer adverse health complaints without a diagnosable condition.

\section{Acknowledgements}

We thank staff and midwives of the Department Obstetrics and Gynaecology at the Komfo Anokye Teaching Hospital, Ghana for their support during the participant recruitment. We also thank laboratory staff of the Department of Molecular Medicine, Kwame Nkrumah University of Science and Technology, Ghana for their support during the laboratory analysis. We acknowledge Professor Youxin Wang for purchasing reagents to support this project.

\section{Conflict of interest}


613 The authors declare that they have no conflict of interest.

614

\section{Funding statement}

616 This work was partially supported by the Australia-China International Collaborative Grant 617 (NH\&MRC-APP1112767-NSFC81561120) and Edith Cowan University (ECU)618 Collaborative Enhancement scheme Round 1 (G1003363). Enoch Odame Anto was supported by ECU-International Postgraduate Research Scholarship.

620

\section{Data availability statement}

622

Data set for this paper is part of a bigger data set from an ongoing Cohort study and is currently stored on internal storage systems of the corresponding author. We are able to provide data specific to this paper on request, once the purpose for the request fits into the ethics approval we received for the work. Request for the data set specific to this paper may be made through the corresponding author. Authors are still be working on the bigger data set to answer other questions and objectives of the bigger study so are unable to make it available to others as at now.

630

Authors Contribution

634

Conceptualization, E.O.A, P.R, D.C, E.A, C.A.T, A.T, Y.W, and W.W.; Methodology, E.O.A, C.A.T and A.T.; Formal Analysis, E.O.A, P.R, D.C, E.A, C.A.T, A.T, Y.W, and W.W.; Investigation, E.O.A, C.A.T and A.T.; Data Curation, EOA; Writing - Original Draft Preparation, E.O.A; Writing - Review \& Editing, E.O.A, P.R, D.C, E.A, C.A.T, A.T, Y.W, 
the final manuscript.

641

642

643

644

645

646

647

648

649

650

651

652

653

654

655

656

657

658

659

660

661

662

663

664

665

666

667

668

669

670

671

672

673

674

675

676

677

678

679

680

681

682

\section{References}

1. Alkema L, Chou D, Hogan D, et al. Global, regional, and national levels and trends in maternal mortality between 1990 and 2015, with scenario-based projections to 2030: a systematic analysis by the UN Maternal Mortality Estimation Inter-Agency Group. Lancet (London, England). 2016 Jan 30;387(10017):462-74.

2. Adua E, Frimpong K, Li X, et al. Emerging issues in public health: a perspective on Ghana's healthcare expenditure, policies and outcomes. The EPMA journal. 2017 Sep;8(3):197-206.

3. Soma-Pillay P, Nelson-Piercy C, Tolppanen $\mathrm{H}$, et al. Physiological changes in pregnancy. Cardiovasc J Afr. 2016;27(2):89.

4. Eick SM, Barrett ES, van 't Erve TJ, et al. Association between prenatal psychological stress and oxidative stress during pregnancy. Paediatric and perinatal epidemiology. 2018 Jul;32(4):318-326.

5. Turpin CA, Sakyi SA, Owiredu WK, et al. Association between adverse pregnancy outcome and imbalance in angiogenic regulators and oxidative stress biomarkers in gestational hypertension and preeclampsia. BMC pregnancy and childbirth. 2015 Aug 25;15:189.

6. Wu F, Tian FJ, Lin Y. Oxidative Stress in Placenta: Health and Diseases. BioMed research international. 2015;2015:293271.

7. Pereira RD, De Long NE, Wang RC, et al. Angiogenesis in the placenta: the role of reactive oxygen species signaling. BioMed research international. 2015;2015:814543.

8. Anto EO, Roberts P, Turpin CA, et al. Oxidative Stress as a Key Signaling Pathway in Placental Angiogenesis Changes in Preeclampsia: Updates in Pathogenesis, Novel Biomarkers and Therapeutics. Current Pharmacogenomics and Personalized Medicine (Formerly Current Pharmacogenomics). 2018;16(3):167-181.

9. Wong W. New connections: The duality of ROS in angiogenesis. Science signaling. 2017 May 16;10(479).

10. Duhig K, Chappell LC, Shennan AH. Oxidative stress in pregnancy and reproduction. Obstet Med. 2016;9(3):113-116.

11. Anto EO, Owiredu WKBA, Sakyi SA, et al. Adverse pregnancy outcomes and imbalance in angiogenic growth mediators and oxidative stress biomarkers is associated with advanced maternal age births: A prospective cohort study in Ghana. PloS one. 2018;13(7):e0200581.

12. Zheng W, Huang W, Zhang L, et al. Changes in Serum Lipid Levels During Pregnancy and Association With Neonatal Outcomes: A Large Cohort Study. Reproductive sciences (Thousand Oaks, Calif). 2018 Sep;25(9):1406-1412.

13. Osawa T. Development and application of oxidative stress biomarkers. Bioscience, biotechnology, and biochemistry. 2018 Apr;82(4):564-572.

14. Owiredu WK, Sakyi SA, Anto EO, et al. Interplay Between Angiogenic Factors and Oxidative Stress Biomarkers in Normal Pregnancy, Gestational Hypertension and Preeclampsia. Medical Journal Obstetrics and Gynecology. 2016;4(3):1086. 
15. de Lucca L, Jantsch LB, Vendrame SA, et al. Longitudinal Study of DeltaAminolevulinate Dehydratase Activity and Oxidative Profile in Healthy Pregnant Women. Biomolecules. 2019 Jan 9;9(1).

16. Golubnitschaja O, Baban B, Boniolo G, et al. Medicine in the early twenty-first century: paradigm and anticipation - EPMA position paper 2016. The EPMA journal. 2016;7:23.

17. Golubnitschaja O, Kinkorova J, Costigliola V. Predictive, Preventive and Personalised Medicine as the hardcore of 'Horizon 2020': EPMA position paper. The EPMA journal. 2014;5(1):6-6.

18. Lemke HU, Golubnitschaja O. Towards personal health care with model-guided medicine: long-term PPPM-related strategies and realisation opportunities within 'Horizon 2020'. The EPMA journal. 2014;5(1):8.

19. Wang W, Russell A, Yan Y. Traditional Chinese medicine and new concepts of predictive, preventive and personalized medicine in diagnosis and treatment of suboptimal health. The EPMA journal. 2014 Feb 13;5(1):4.

20. Golubnitschaja O. Time for new guidelines in advanced diabetes care: Paradigm change from delayed interventional approach to predictive, preventive \& personalized medicine. The EPMA journal. 2010 Mar;1(1):3-12.

21. Yan Y-X, Liu Y-Q, Li M, et al. Development and evaluation of a questionnaire for measuring suboptimal health status in urban Chinese. Journal of Epidemiology. 2009;19(6):333-341.

22. Wang W, Yan Y. Suboptimal health: a new health dimension for translational medicine. Clinical and translational medicine. 2012 Nov 14;1(1):28.

23. Adua E, Roberts P, Wang W. Incorporation of suboptimal health status as a potential risk assessment for type II diabetes mellitus: a case-control study in a Ghanaian population. The EPMA journal. 2017 Dec;8(4):345-355.

24. Kupaev V, Borisov O, Marutina E, et al. Integration of suboptimal health status and endothelial dysfunction as a new aspect for risk evaluation of cardiovascular disease. The EPMA journal. 2016;7(1):19.

25. Ge S, Xu X, Zhang J, et al. Suboptimal health status as an independent risk factor for type 2 diabetes mellitus in a community-based cohort: the China suboptimal health cohort study. The EPMA journal. 2019 Mar;10(1):65-72.

26. Wang Y, Liu X, Qiu J, et al. Association between Ideal Cardiovascular Health Metrics and Suboptimal Health Status in Chinese Population. Scientific reports. 2017 Nov 3;7(1):14975.

27. Yan YX, Dong J, Liu YQ, et al. Association of suboptimal health status and cardiovascular risk factors in urban Chinese workers. Journal of urban health : bulletin of the New York Academy of Medicine. 2012 Apr;89(2):329-38.

28. Yan YX, Dong J, Liu YQ, et al. Association of suboptimal health status with psychosocial stress, plasma cortisol and mRNA expression of glucocorticoid receptor alpha/beta in lymphocyte. Stress (Amsterdam, Netherlands). 2015 Jan;18(1):29-34.

29. Alzain MA, Asweto CO, Zhang J, et al. Telomere Length and Accelerated Biological Aging in the China Suboptimal Health Cohort: A Case-Control Study. Omics : a journal of integrative biology. 2017 Jun;21(6):333-339.

30. Anto EO, Roberts P, Coall D, et al. Integration of suboptimal health status evaluation as a criterion for prediction of preeclampsia is strongly recommended for healthcare management in pregnancy: a prospective cohort study in a Ghanaian population [journal article]. EPMA Journal. 2019 August 05;10(3):211-226.

31. Wang Y, Ge S, Yan Y, et al. China suboptimal health cohort study: rationale, design and baseline characteristics. Journal of translational medicine. 2016 Oct 13;14(1):291. 
32. Benzie IF, Strain JJ. The ferric reducing ability of plasma (FRAP) as a measure of "antioxidant power": the FRAP assay. Analytical biochemistry. 1996 Jul 15;239(1):70-6.

33. Cervantes Gracia K, Llanas-Cornejo D, Husi H. CVD and Oxidative Stress. J Clin Med. 2017;6(2):22.

34. Cervantes Gracia K, Llanas-Cornejo D, Husi H. CVD and Oxidative Stress. J Clin Med. 2017 Feb 20;6(2).

35. Kostov K, Halacheva L. Role of Magnesium Deficiency in Promoting Atherosclerosis, Endothelial Dysfunction, and Arterial Stiffening as Risk Factors for Hypertension. International journal of molecular sciences. 2018 Jun 11;19(6).

36. Wolf FI, Trapani V, Simonacci M, et al. Magnesium deficiency and endothelial dysfunction: is oxidative stress involved? Magnesium research. 2008 Mar;21(1):5864.

37. Zheltova AA, Kharitonova MV, Iezhitsa IN, et al. Magnesium deficiency and oxidative stress: an update. Biomedicine (Taipei). 2016;6(4):20-20.

38. Lopes van Balen VA, van Gansewinkel TAG, de Haas S, et al. Physiological adaptation of endothelial function to pregnancy: systematic review and meta-analysis. Ultrasound in obstetrics \& gynecology : the official journal of the International Society of Ultrasound in Obstetrics and Gynecology. 2017 Dec;50(6):697-708.

39. Sakyi SA, Owiredu WK, Anto EO, et al. Individual and Combined Diagnostic Accuracy of Biochemical Markers for Detecting Early On-Set Preeclampsia. SOJ Gynecology, Obstetrics \& Women's Health 2016;2(1):9. 